ARTICLE

DOI: $10.1038 / \mathrm{s} 41467-018-05550-5$

\title{
Hypermethylation of gene body CpG islands predicts high dosage of functional oncogenes in liver cancer
}

Maria Arechederra1, Fabrice Daian (10 1, Annie Yim², Sehrish K. Bazai', Sylvie Richelme ${ }^{1}$, Rosanna Dono ${ }^{1}$ Andrew J. Saurin (10 ${ }^{1}$, Bianca H. Habermann (1) ${ }^{1}$ \& Flavio Maina (i) ${ }^{1}$

Epigenetic modifications such as aberrant DNA methylation reshape the gene expression repertoire in cancer. Here, we used a clinically relevant hepatocellular carcinoma (HCC) mouse model (Alb-R26 $\left.6^{M e t}\right)$ to explore the impact of DNA methylation on transcriptional switches associated with tumorigenesis. We identified a striking enrichment in genes simultaneously hypermethylated in CpG islands (CGIs) and overexpressed. These hypermethylated CGIs are located either in the $5^{\prime}$-UTR or in the gene body region. Remarkably, such CGI hypermethylation accompanied by gene upregulation also occurs in $56 \%$ of HCC patients, which belong to the "HCC proliferative-progenitor" subclass. Most of the genes upregulated and with hypermethylated CGIs in the Alb-R26 Met HCC model undergo the same change in a large proportion of HCC patients. Among reprogrammed genes, several are well-known oncogenes. For others not previously linked to cancer, we demonstrate here their action together as an "oncogene module". Thus, hypermethylation of gene body CGls is predictive of elevated oncogene levels in cancer, offering a novel stratification strategy and perspectives to normalise cancer gene dosages.

\footnotetext{
${ }^{1}$ Aix Marseille Univ, CNRS, Developmental Biology Institute of Marseille (IBDM), Parc Scientifique de Luminy, Aix Marseille Univ, 13009 Marseille, France. ${ }^{2}$ Computational Biology Group, Max Planck Institute of Biochemistry, 82152 Martinsried, Germany. Correspondence and requests for materials should be addressed to F.M. (email: flavio.maina@univ-amu.fr)
} 
A ppropriate timing and dosage of gene expression in healthy cells is ensured by complex processes integrating genetic and epigenetic information. Alterations of these mechanisms are frequent in cancer and underline functional changes in genes acting as oncogenes or tumour suppressors ${ }^{1-3}$. The use of high-throughput sequencing has contributed considerably to our understanding on how epigenetic modifications switch genomic regions from an inaccessible closed conformation to an open state-and vice-versa-contributing to changes in the transcriptome landscape ${ }^{4-6}$. DNA methylation is an essential epigenetic mechanism influencing gene expression levels in cells and alterations lead to dramatic changes in malignant cells. The cancer landscape is generally characterised by a diffuse DNA hypomethylation and by focal hypermethylation in CpG-rich regions known as $\mathrm{CpG}$ islands (CGIs) ${ }^{1,7}$. CGI hypermethylation at promoters represses transcription of genes acting as tumour suppressors, a well-known mechanism operating in cancer ${ }^{8}$. However, DNA methylation at intergenic regions and gene bodies is gaining relevance for its impact on gene expression ${ }^{9,10}$. Aberrant DNA methylation of large clusters of transcriptional enhancers, known as super-enhancers, leads to dramatic transcriptional changes of gene sets in cancer ${ }^{11}$. A large fraction of DNA methylation is also observed in gene body CGIs, with an apparent intriguing positive correlation between methylation and gene expression ${ }^{12,13}$. Such contradiction on DNA methylation effects in promoter versus gene body CGIs remains poorly understood.

The relevance of epigenetics in tumorigenesis has been further emphasised through recent large-scale screen analyses focused on cancer patients carrying either histone mutations or alterations in genes regulating DNA methylation-histone modifications ${ }^{2}$. Results from these studies highlighted how such mutations dramatically modify the epigenetic and gene expression landscapes. For example, aberrant DNA methylation has been recently reported in acute myeloid leukaemia patients with DNMT3A mutations ${ }^{14}$. Abnormal recruitment of PRC2 complex and DNA methylation occurs in paediatric glioblastoma with Histone $\mathrm{H} 3$ mutant variants ${ }^{15}$. Gene expression changes caused by histone H3K36 mutation is associated with sarcomagenesis ${ }^{16}$. Nevertheless, the epigenetic reshape occurs also in the absence of specific mutations in chromatin modulators ${ }^{17}$. It is the case of classical oncogenes and tumour suppressors, which can trigger profound chromatin alterations with consequences on gene expression $^{18,19}$. For example, an oncogenic splice variant of EGFR leads to genome-wide activation of putative enhancers in glioblastoma $^{20}$. Oncogenic EGFR leads to DNA methylationmediated transcriptional silencing of tumour suppressors in lung cancer and glioblastoma ${ }^{21}$. Deregulated Ras signalling reshapes the enhancer landscape leading to aberrant oncogene expression $^{22}$. PI3K/Akt pathway activation induces promoterassociated gene activation in breast cancer ${ }^{23}$. Overall, such screen approaches have also contributed to identify new genes, whose functional relevance in cancer was previously unknown and/or which deregulations can be used as cancer biomarkers for prognosis/patient stratification.

We recently reported a cancer mouse model in which slight increases in wild-type Met receptor tyrosine kinase (RTK) levels in the liver are sufficient for spontaneous tumours in mice (Alb$\left.R 26^{M e t}\right)$. These genetic studies conceptually illustrate how the shift from physiological to pathological conditions results from perturbations in subtle signalling dosage. Through gene expression analysis, the $A l b-R 26^{M e t}$ mice were shown to model a HCC patient subgroup corresponding to the so-called "proliferativeprogenitor" subclass ${ }^{24}$, demonstrating the clinical relevance of this genetic system. The uniqueness of this genetic system was also illustrated by its usefulness to identify new synthetic lethal interactions as potential therapies for HCC subgroups ${ }^{24}$. Here, we employed the Alb-R26 ${ }^{\mathrm{Met}}$ cancer model for integrative genomewide studies combining methylome and transcriptome outcomes and compared them with those from HCC patients. Results show an enrichment in genes overexpressed and with hypermethylated CGI, with expression levels positively correlating with the CGI distance to the ATG. Whereas most of the upregulated genes are well-known oncogenes, the implication of others in cell tumorigenic properties is demonstrated here through functional studies. Enrichment of genes both overexpressed and with hypermethylated CGIs characterises the "proliferative-progenitor" HCC patient subset, which is modelled by the Alb-R26 ${ }^{\text {Met }}$ genetic system. Collectively, these results show that an epigenetic reprogramming process ensuring increased dosage of an "oncogenic module" involving multiple genes operates in tumorigenesis.

\section{Results}

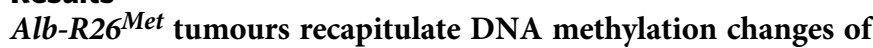
HCC patient subgroups. We recently showed how the Alb$R 26^{M e t}$ genetic system is a unique tool to model: (a) the tumorigenic program, (b) the "proliferative-progenitor" HCC patient subgroup and (c) functionality of signalling alteration for drug discovery ${ }^{24}$. For its use to study the contribution of epigenetic modifications linked to cancer, we reasoned that it was first necessary to determine whether the $A l b-R 26^{M e t}$ tumorigenesis occurs in a stable genomic context or is associated with chromosomal deletions/duplications. Comparative genomic hybridisation analyses on DNA inputs from $16 \mathrm{Alb}-\mathrm{R} 26^{\mathrm{Met}}$ tumours and 8 control livers excluded chromosomal instability (Supplementary Fig. 1). These findings therefore reinforce the appropriateness of the $A l b-R 26^{M e t}$ cancer model as a relevant genetic system to study the epigenetic reprogramming associated with cancer, which we addressed by bioinformatically integrating data from methylome and transcriptome screens (Fig. 1a).

DNA methylation changes were scored by performing MethylMiniSeq EpiQuest sequencing on $10 \mathrm{Alb}-\mathrm{R} 26^{\mathrm{Met}}$ tumours (previously histologically identified as $\mathrm{HCC}^{24}$ ) and 3 control livers (Supplementary Fig. 2A). Mean methylation levels were modestly, yet significantly, different across all measured CpGs $(P$ value $=2.4 \mathrm{E}-03$; Fig. $1 \mathrm{~b})$, being able to group tumours and controls into two distinct clusters (Fig. 1c). A remarkable predominance of global hypomethylation was observed in tumours compared with livers (Fig. 1b, Supplementary Fig. 2B). Accordingly, we observed an enrichment in hypomethylated CpGs located outside CGIs ( $P$-value $=3 \mathrm{E}-04$; Fig. 1b, Supplementary Fig. 2C). In contrast, a significant enrichment of hypermethylation at $\mathrm{CpGs}$ located within CGIs characterised Alb-R26 $6^{\text {Met }}$ tumours compared with control livers $(P$-value $=$ 3.9E-03; Fig. 1b, d, Supplementary Fig. 2D). These traits of CpG methylation changes, according to the $\mathrm{CpG}$ location with respect to CGIs, are consistent with those largely reported in the literature ${ }^{1}$. Focusing on differentially methylated CpGs located at annotated CGIs, we identified 513 CGIs with a $\beta$-value methylation difference of \pm 0.2 and a false discovery rate (FDR) $<0.05$ (Fig. 1d, Supplementary Fig. 2D, Supplementary Data 1). These CGIs were homogeneously distributed amongst all 19 autosomal and 1 sex chromosome mouse pairs (Supplementary Fig. 2E). Among CGIs with differentially methylated CpGs, $82 \%$ were hypermethylated in $A l b-R 26^{M e t}$ HCC compared to controls (Fig. 1d).

To explore the relevance of these methylation changes in the context of human HCC disease, we used genome-wide DNA methylation data from a cohort of 41 HCC patients, for which data are available for both: (a) methylation and expression; (b) 
a
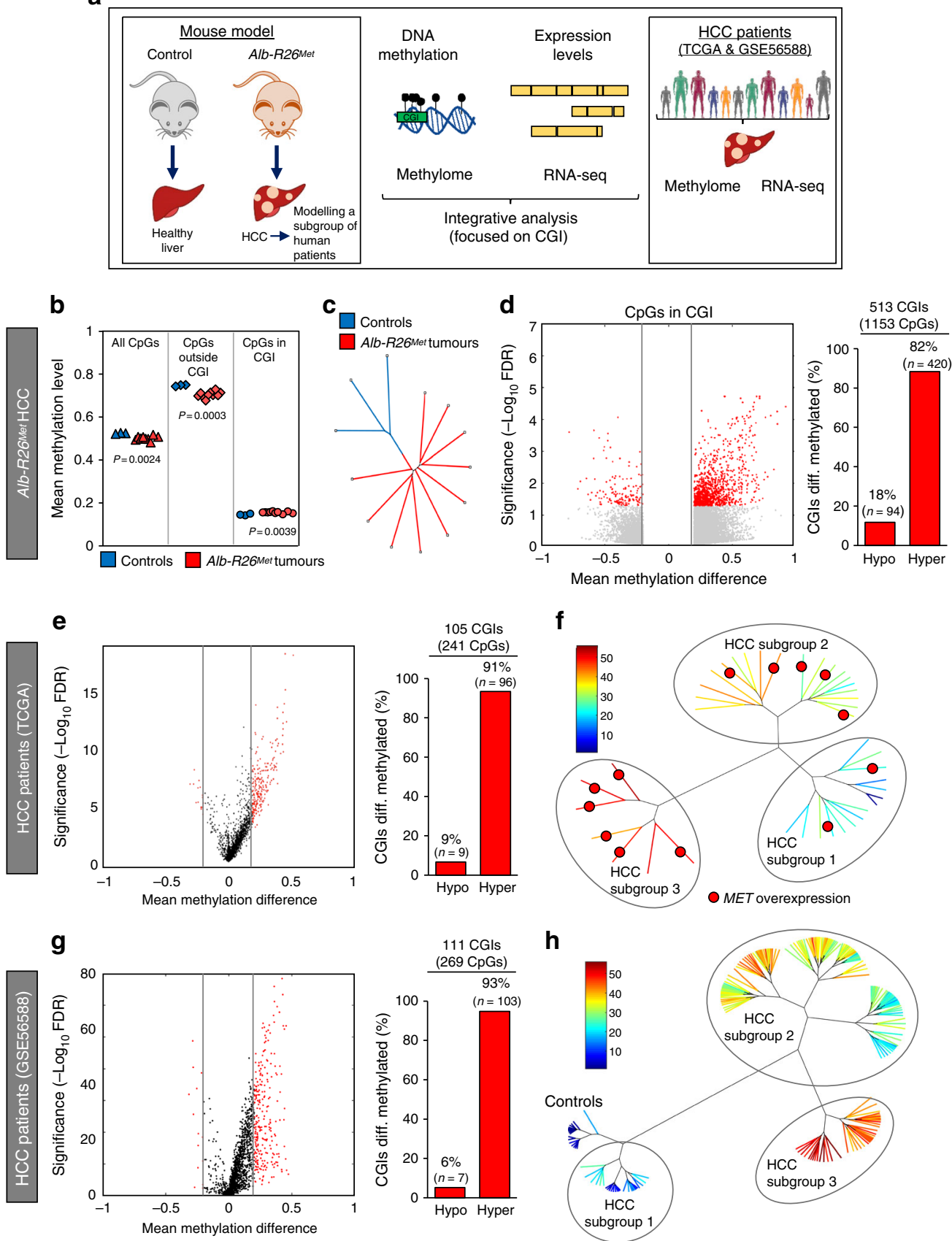

Fig. 1 Methylome studies identify an enrichment of CGI hypermethylation in Alb-R26 Met tumours, also present in a subset of HCC patients. a Schematic representation of the overall strategy employed. DNA methylation and gene expression levels were analysed in Alb-R26Met tumours and control livers. Outcomes were compared with HCC human database. b Mean methylation levels in controls and Alb-R26 Met tumours, focusing on all CpGs, CpGs outside CGls and CpGs in CGls. c Unrooted distance tree using the overall DNA methylation content subdivides Alb-R26 Met tumours and controls in two distinct clusters. d Volcano plot reporting methylation differences with significance (expressed as - Log 10 FDR) for CpGs in CGIs in Alb-R26Met tumours versus control (left). Significant differences (methylation difference $>0.2$ and FDR $<0.05$ ) are shown in red. Graph reporting the percentage (and numbers) of hypomethylated versus hypermethylated CGIs (right). e Volcano plot reporting the mean methylation differences with significance (expressed as - Log 10 FDR) in HCC patients from TCGA (left) for differentially methylated CGls identified in Alb-R26 Met tumours. Similar methylation levels in HCC patients and controls are reported in black, whereas changes $(>0.2)$ are reported in red. On the right, the graph reports the percentage (and numbers) of hypomethylated versus hypermethylated CGIs. f Unrooted distance tree of the 41 TCGA HCC patients showing patient segregation in three distinct subgroups, according to the $416 \mathrm{CGls}$ found differentially methylated in Alb-R26 Met tumours. Red dots highlight patients in which MET is overexpressed $\left(\log _{2} \mathrm{FC}>1\right)$. Patients are reported in different colours according to the percentage of overlap (the scale in percentage is shown on the left). Note the striking correlation between differentially methylated CGIs and MET overexpression in the HCC patient subgroup 3. $\mathbf{g}$, $\mathbf{h}$ Volcano plot (g) and unrooted distance tree (h) from studies using a second cohort of $224 \mathrm{HCC}$ patients and 10 controls (GSE56588 dataset) 
tumour and adjacent liver as control (from The Cancer Genomic Atlas; $\mathrm{TCGA}^{25}$ ). For comparisons between mouse and human data, we first mapped the 513 identified mouse CGIs (mm9) to the corresponding CGIs in human (hg19), using the UCSC toolbox. 501 out of 513 CGIs were successfully matched between the two genomes. For 416 CGIs, human methylome data were available in TCGA dataset (Supplementary Fig. 3A; Supplementary Data 2). We extracted the methylation $\beta$-value for the CpGs within these human CGIs and calculated the mean methylation difference for each $\mathrm{CpG}$ and for each of the 41 HCC patients. While the majority of these CpGs showed a methylation difference below 0.2 , a proportion of CGIs (24\%) were differentially methylated (FDR $<0.05)$ with a hypermethylation enrichment score similar to the Alb-R26 $6^{\text {Met }}$ HCC (91\%; Fig. 1e, Supplementary Fig. 3B). As the analysed cohort includes patients with widely diverse aetiologies and characteristics, we next analysed the 416 CGIs in the individual patients. Intriguingly, hierarchical clustering analysis segregated these HCC patients into three distinct subgroups, with one subgroup composed of seven patients reaching $43-56 \%$ overlap with the Alb-R26 $6^{\text {Met }}$ list (subgroup-3; Fig. 1f, Supplementary Fig. 3C). The relevance of the Alb-R26 $6^{\text {Met }}$ methylation changes in the context of human HCC was further assessed in a second distinct cohort of 234 human samples (224 HCC patients and 10 control individuals ${ }^{26}$ ). $27 \%$ of CGIs differentially methylated in Alb-R26 $6^{\text {Met }} \mathrm{HCC}$ are also altered in human HCCs, again with an enrichment in hypermethylation (93\%; Fig. 1g, Supplementary Fig. 4A). Moreover, these methylation changes distinguished controls from HCC patients, which further segregate into three subgroups. HCC subgroup- 3 reaches about $50 \%$ CGI overlap with the Alb-R26 list (Fig. 1h, Supplementary Fig. 4B).

Next, we asked whether there would be any correlation between MET alterations with the three human HCC subgroups identified by the Alb-R26 ${ }^{M e t}$ methylome screening. Concerning the HCC patient cohort from TCGA, we were able to perform correlative studies as RNA-seq and mutation data are available. In particular, we analysed MET mutations and MET expression levels for each patient belonging to the 3 different HCC subgroups. All HCC patients carry the wild-type form of MET, which is in agreement with rare mutations of MET in HCC. Concerning expression levels, MET is overexpressed in 86\% (6/7) of HCC patients belonging to subgroup-3 (which best overlaps with CGI methylation changes in Alb-R26 ${ }^{\text {Met }}$ ), in $32 \%(6 / 19)$ to HCC subgroup-2, and only in 13\% (2/15) to HCC subgroup-1 (Fig. 1f; patients with $M E T$ overexpression are highlighted with a red dot; Supplementary Fig. 3C-F). For the HCC patient cohort from GSE56588, expression data (array) are only available for some patients and without information about mutations. Therefore, correlative studies were not possible with this HCC cohort. Together, these findings show that liver cancer modelled by the Alb-R26 $6^{\text {Met }}$ genetic system is characterised by methylation changes of specific CGIs, with a predominant hypermethylation profile. A high proportion of these alterations are also found in HCC patient subgroups. Furthermore, there is a striking correlation between differentially methylated CGIs and MET overexpression in the HCC patient subgroup modelled by the Alb-R26 $6^{\text {Met }}$ genetic setting.

Enrichment in CGI hypermethylation is necessary for Alb$\mathbf{R 2 6}^{\text {Met }}$ tumorigenesis. The overall enrichment in CGI hypermethylation in the Alb-R26 ${ }^{\text {Met }}$ genetic system prompted us to determine its relevance for cell tumorigenic properties. We designed different demethylating experimental conditions using low doses of Decitabine ( $0.3 \mu \mathrm{M}$; Fig. 2a), according to previously reported protocols ${ }^{12}$. We used three different Alb-R26 ${ }^{\text {Met }}$ HCC cell lines, established from individual Alb-R26 $6^{\text {Met }}$ tumours ${ }^{24}$. Decitabine treatment does not affect cell viability of $A l b-R 26^{\mathrm{Met}}$ HCC cells, as well as of MLP-29 cells, a mouse liver progenitor cell line that is not tumorigenic as illustrated by its inability to form colonies in anchorage-independent growth assays (Fig. 2b). Instead, Decitabine treatment interferes with Alb-R26 $6^{\text {Met }}$ cell tumorigenic properties, irrespective of the HCC cell line used, as exemplified by: (a) reduced colony numbers when cells are grown in an anchorage-independent manner (Fig. 2c); (b) reduced number and size of foci when cells are grown in an anchoragedependent manner (Fig. 2d); (c) reduced tumour spheres when cells are grown in self-renewal conditions (Fig. 2e). The effect of global demethylation on cell tumorigenicity was further analysed in vivo by performing xenografts in nude mice. The tumour volume was significantly reduced in mice either injected with Decitabine pre-treated Alb-R26 ${ }^{\text {Met }} \mathrm{HCC}$ cells or when Decitabine pulses were administered to mice injected with untreated $A l b$ $R 26^{\text {Met }}$ HCC cells (Fig. 2f-left). Decitabine doses used in vivo were not toxic, as revealed by no significant changes on the mouse weight during the treatment (Fig. 2f-right). Together, these results indicate that the overall enrichment in CGI hypermethylation is functionally relevant for tumorigenesis modelled by the Alb-R26 $6^{\text {Met }}$ genetic system.

CGI hypermethylation correlates with gene upregulation in Alb-R26 ${ }^{\text {Met }}$ HCC. Alterations in DNA methylation are known to impact gene expression. We analysed the expression levels of the 431 genes with differentially methylated CGIs in Alb-R26 tumours using high-coverage RNA-seq data (4 Alb-R26 $6^{\text {Met }}$ tumours and 4 control livers). Studies highlighted 93 genes differentially expressed $\left(\log _{2} \mathrm{FC}>1, \quad \mathrm{FDR}<0.05\right.$; Supplementary Data 3). According to the Kyoto Encyclopaedia of Genes and Genomes (KEGG) database, several cancer-related pathways were significantly enriched, such as MAPK signalling, viral carcinogenesis, pathways in cancer, TGF- $\beta$ signalling, cell cycle, renal cell carcinoma (Fig. 3a, Supplementary Fig. 5), strengthening the significance of genes differentially methylated and expressed in the Alb-R26 $6^{\text {Met }}$ cancer model. Remarkably, the top-ranked MAPK pathway is coherent with its essential functionality for $A l b-R 26^{\mathrm{Met}}$ tumorigenicity, as previously reported ${ }^{24}$. Among genes differentially methylated and expressed, 36 genes showed the expected inverse correlation between methylation and expression where 20 genes are hypomethylated and overexpressed, and 16 genes are hypermethylation and downregulated (Fig. 3b, Supplementary Data 3). Unexpectedly, 55 genes (59\%) were found hypermethylated and overexpressed (Fig. 3b). Thus, tumorigenesis modelled by the Alb-R26 ${ }^{\mathrm{Met}}$ mice is characterised by a set of genes with changes in CGI methylation accompanied by a reprograming of transcript levels.

The intriguing enrichment in hypermethylated and overexpressed genes drove us to analyse the position of the hypermethylated CGIs with respect to the ATG. Interestingly, the CGI of overexpressed genes is either close to the ATG or in the gene body region, in contrast to the CGI position of downregulated genes exclusively located around the ATG (Supplementary Fig. 6). Concerning the 55 genes hypermethylated and overexpressed, they can be subdivided into two groups. Group-I includes 31 genes, for which the CGIs are located between $-50 \%$ and $30 \%$ relative to the ATG (predominantly into the 5'-UTR). Group-II includes 24 genes, whose CGIs are located much further from the ATG (from $30 \%$ of the gene body relative to the ATG to the transcription termination site), corresponding to gene body regions (Fig. 3c, Supplementary Fig. S6). Next, we analysed whether the CGI location influences gene expression. Intriguingly, the extent of overexpression is significantly higher 
a

\begin{tabular}{l} 
No treatment \\
$\square \square \quad \square \square \square \square \square \square \square \square \square \square \square \square \square \square$ \\
Decitabine pre-treatment \\
$\square \square$ Decitabine pre-treatment + pulses \\
$\square \square \quad \square \square \square \square \square \square \square \square \square \square \square \square \square \square \square$ \\
\hline No treatment $\square \square \square \square \square \square \square \square \square \square \square \square \square \square \square$ \\
Decitabine1 pulse \\
$\square \square$ in $\square \square \square \square \square \square \square \square \square \square \square \square \square \square$ \\
$\square \square$ Decitabine 3 pulses $\square \square \square \square \square \square \square \square \square \square \square \square \square$
\end{tabular}
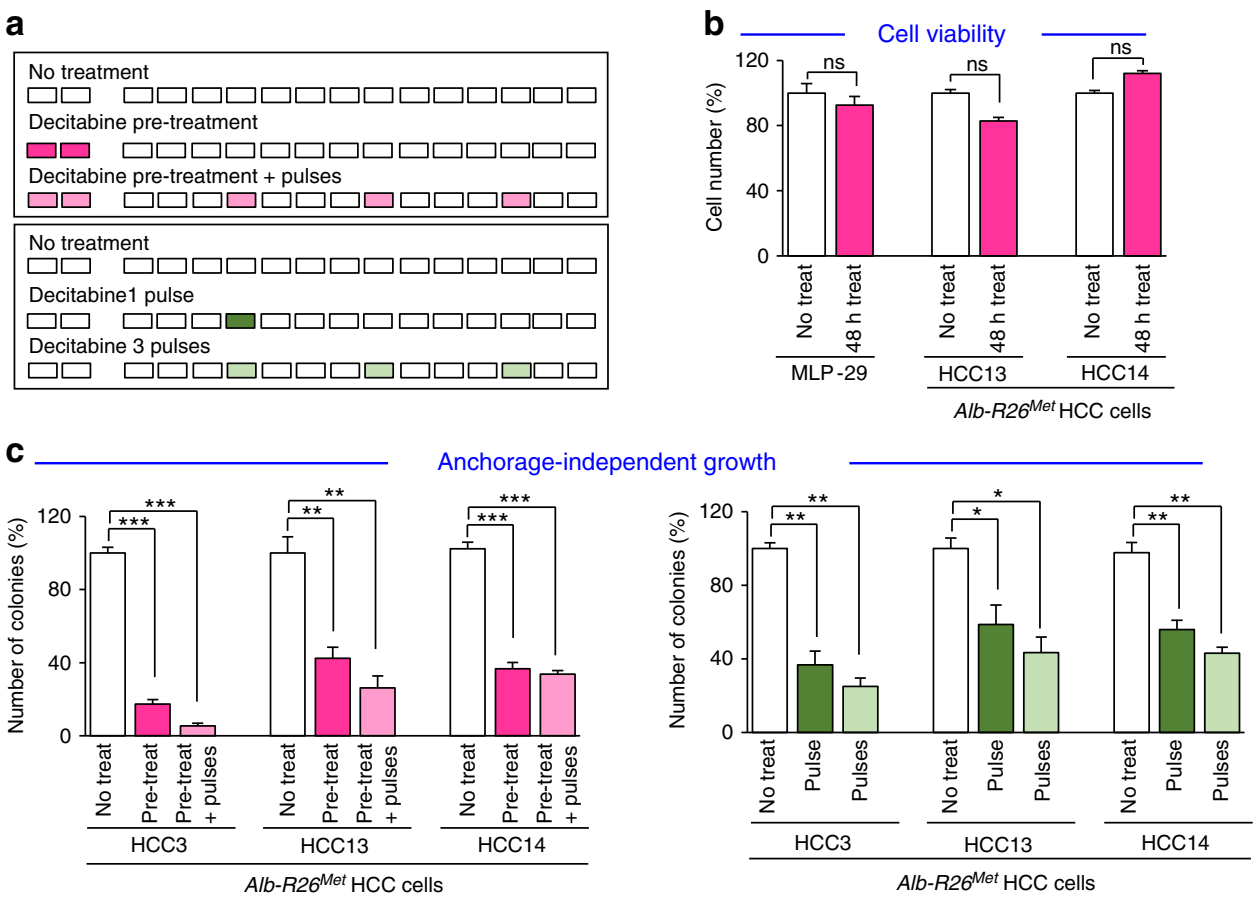

d
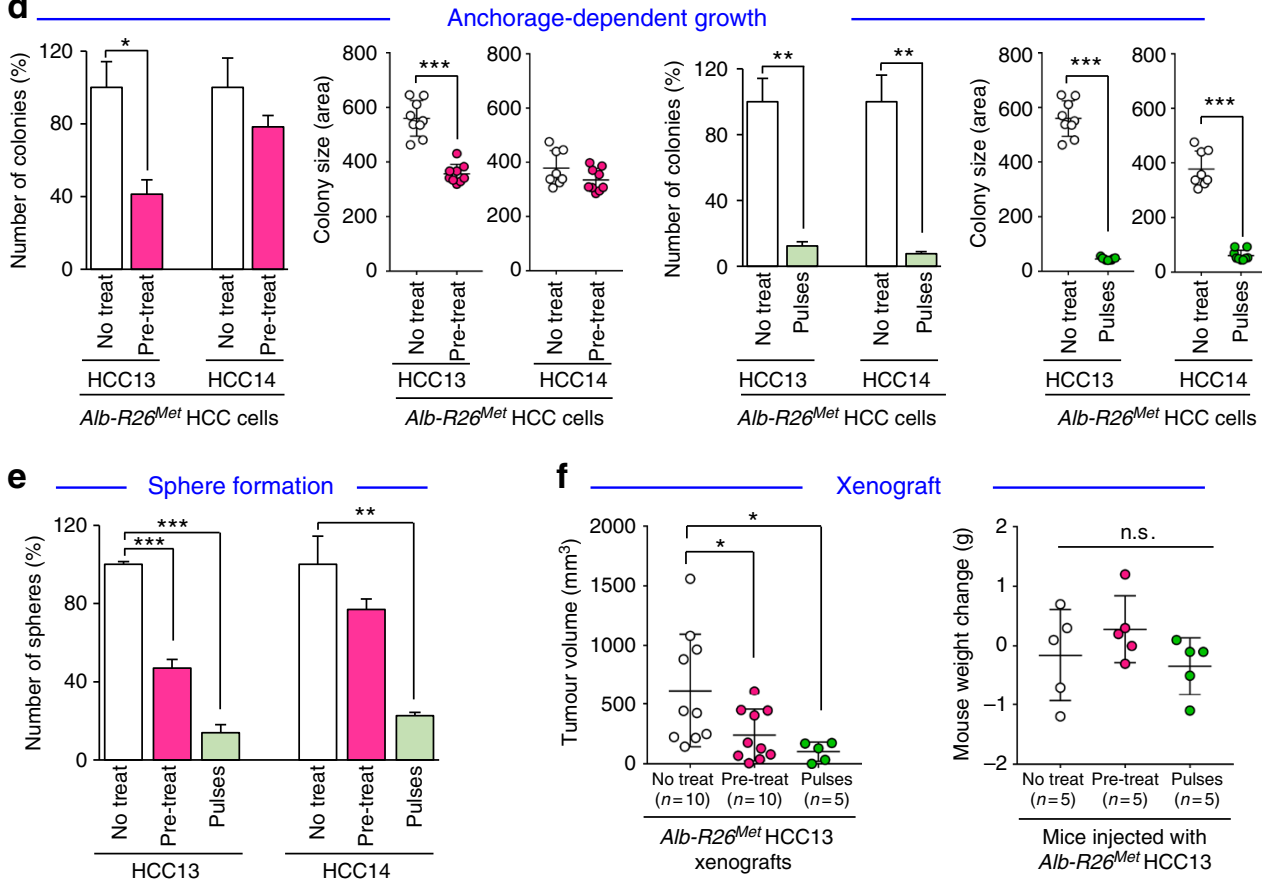

Fig. 2 Global CGI hypermethylation is functionally relevant for Alb-R26 Met tumorigenesis. a Scheme reporting demethylating treatments (Decitabine; $0.3 \mu \mathrm{M})$ used for in vitro and in vivo experiments with Alb-R26 Met $\mathrm{HCC}$ cells. Cells were pre-treated (48 h) before using them for experiments with or without subsequent Decitabine pulses every 3 days (top; pink). Untreated cells were exposed to pulses of demethylating treatments during the assay (bottom; green). b Effect of Decitabine treatment ( $48 \mathrm{~h}$ ) on cell viability of mouse liver progenitor MLP-29 cells and of Alb-R26Met HCC cells (HCC13 and HCC14). c Anchorage-independent growth (soft agar) assay using 3 different Alb-R26 Met HCC cell lines (HCC3, 13 and 14) showing effects of demethylating treatments described in $\mathbf{a}$. $\mathbf{d}$ Effect of Decitabine treatment in anchorage-dependent growth (foci formation) assay of 2 different Alb-R26Met HCC cell lines (HCC13 and 14). Graphs report number and size of colonies. e Decitabine (pre-treatment and treatment) reduces numbers of tumour spheres derived from Alb-R26 Met HCC cells ( $\mathrm{HCC13}$ and HCC14). $\mathbf{f}$ Left: Graph reporting the tumour volume of mice injected either with untreated cells (No treat), with Decitabine pre-treated Alb-R26 Met HCC cells (Pre-treat), or with untreated cells following pulses of Decitabine in vivo treatments (Pulses). Note that tumour volume was significantly reduced in mice injected either with Decitabine pre-treated Alb-R26 Met HCC cells or with untreated cells following in vivo Decitabine pulses. Right: Mouse weight of the indicated groups was measured before and after xenograft experiments. No significant changes were observed, indicating that the dose of Decitabine used in vivo was not toxic. Significant differences between groups are indicated on the top. Not significant (ns): $P>0.05,{ }^{\star} P<0.05,{ }^{\star \star} P<0.01 ;{ }^{\star \star \star} P<0.001$ 
a

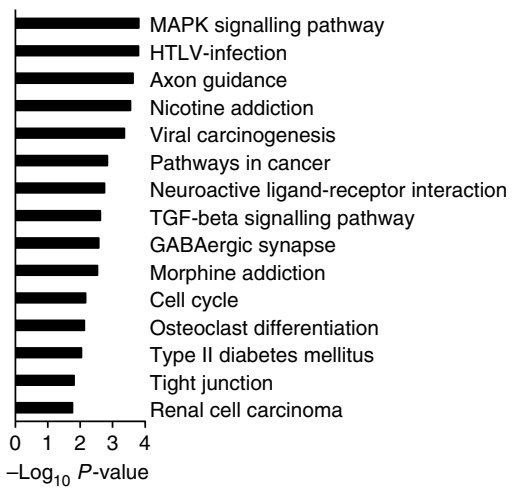

C

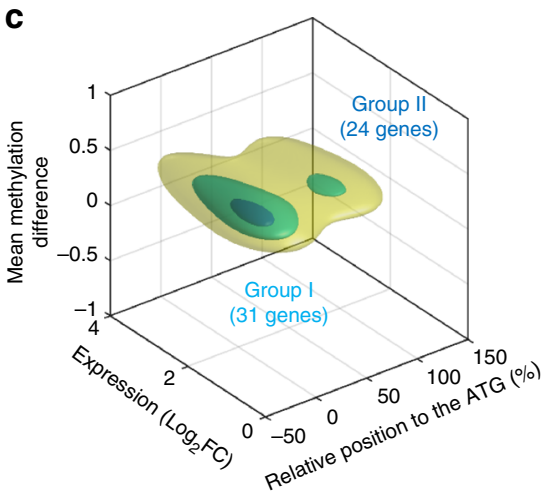

b

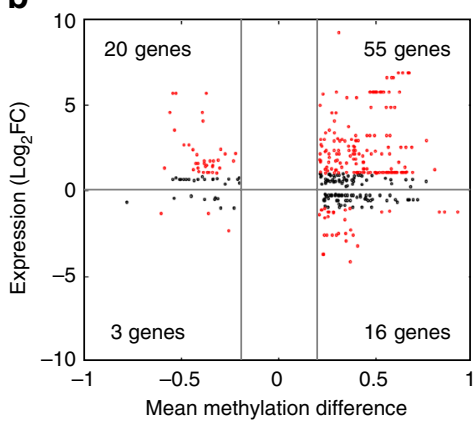

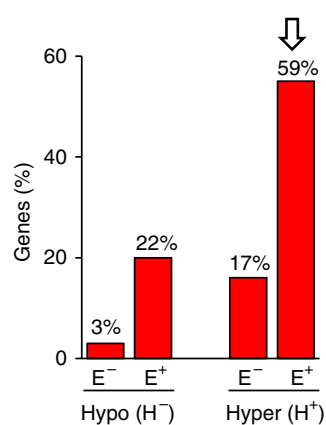

d

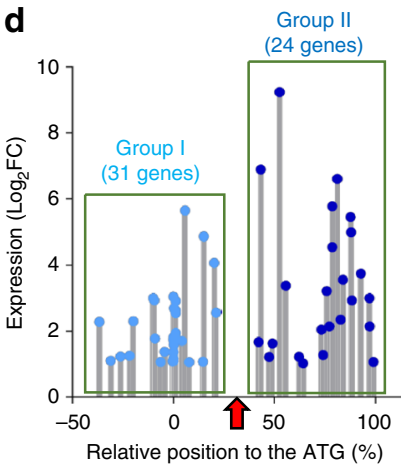

$\mathbf{e}$

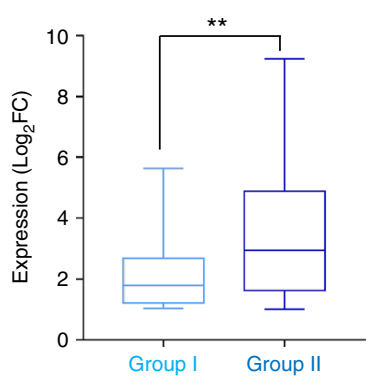

f

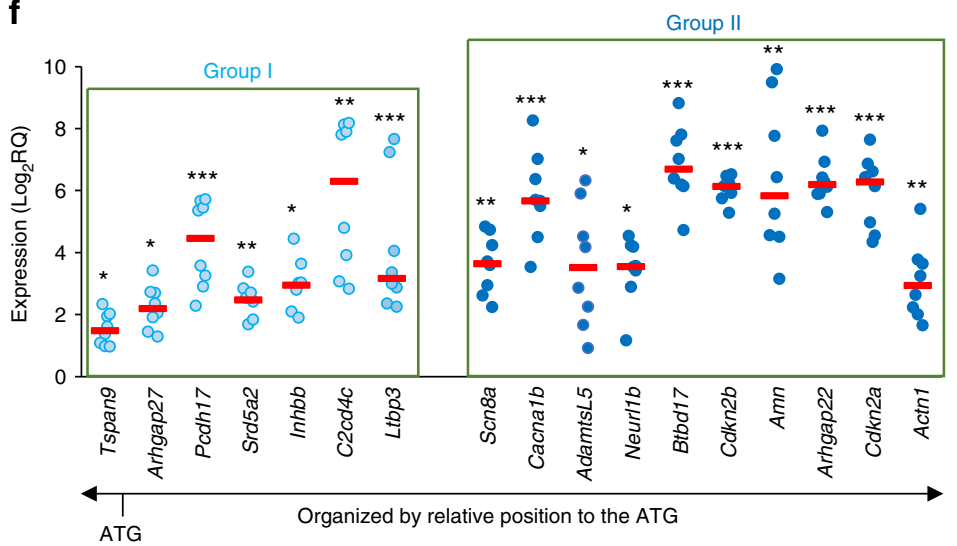

g

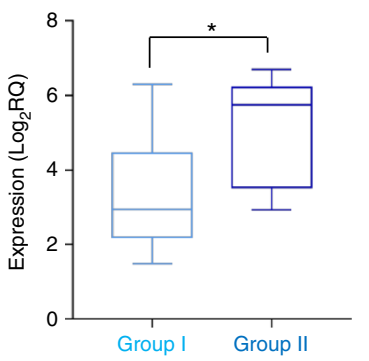

Fig. $3 \mathrm{Alb}-R 26^{\mathrm{Met}}$ tumours are characterised by an enrichment in genes overexpressed and with hypermethylated CGIs. a Histogram reporting the KEGG pathway enrichment analysis for genes with changes in CGI methylation and expression in Alb-R26 Met tumours, ordered according to the - Log $_{10} P$-value. b Left: Methylation differences versus expression for all genes with CGls hypermethylated $\left(\mathrm{H}^{+}\right)$or hypomethylated $\left(\mathrm{H}^{-}\right)$in $A l b-R 26^{M e t}$ tumours.

Expression values are relative to controls. Dots correspond to single differentially methylated $\mathrm{CpG}$ and the corresponding gene expression (genes which expression is significantly below or above $\log _{2} \mathrm{FC} \pm 1$ are indicated in red). Right: Graph reporting the percentage of downregulated ( $E^{-}$) and upregulated ( $E$ $\left.{ }^{+}\right)$genes among those with a hypomethylated $\left(\mathrm{H}^{-}\right)$or hypermethylated $\left(\mathrm{H}^{+}\right) \mathrm{CGI}$. Note the enrichment of genes overexpressed and with hypermethylated CGls (indicated by an arrow), on which subsequent studies were focused. c For the 55 genes overexpressed and with hypermethylated CGI in Alb-R26 $6^{M e t}$ tumours, 3D density plot shows their distribution according to relative position to the ATG (as percentage), gene expression level (as Log ${ }_{2}$ FC) and CGI methylation (as $\beta$-value difference). Note that genes segregate into two groups, according to their relative position to the ATG. d Graph reporting the individual expression level (as $\log _{2} \mathrm{FC}$; from RNA-seq data) of hypermethylated and overexpressed genes found in Alb-R26 Met tumours compare to controls. Note that the relative position of the hypermethylated CGI to the ATG well segregate the two groups (indicated with a red arrow). e Box plot illustrating the global expression levels of genes in Group-I and Group-II. $\mathbf{f}$ Graph reporting individual expression levels (as Log 2 FC; by RT-qPCR) of genes belonging to the two groups in Alb-R26 Met tumours $(n=8)$ relative to control livers $(n=6)$. Red lines report the median Log ${ }_{2} \mathrm{FC}$ in expression. Note that genes are distributed according to the location of the hypermethylated CGI relative to the ATG. $\mathbf{g}$ Box plot showing the global Log ${ }_{2} \mathrm{FC}$ in expression (according to data in F) of genes in Group-I and Group-II. In $\mathbf{e}$ and $\mathbf{g}$, the median is reported by a line and bars extend to the minimum/maximum values. Significance is indicated on the top. ${ }^{\star} P<0.05,{ }^{\star \star} P<0.01,{ }^{\star \star \star} P<0.001$ 
for Group-II than Group-I (median $\log _{2} \mathrm{FC}=2.15 \pm$ versus median $\log _{2} \mathrm{FC}=3.38 \pm 0.4$; Fig. $3 \mathrm{~d}$, e). Importantly, the promoter CGI methylation status of genes belonging to Group-II was similar in Alb-R26 ${ }^{\text {Met }}$ tumours and control livers, thus excluding that changes in promoter methylation could influence gene expression levels (Supplementary Data 4). We corroborated these results through RT-qPCR analysis of a subset of genes belonging to both groups in Alb-R26 $6^{M e t}$ tumours $(n=8)$ relative to control livers $(n=6)$. Results showed consistent upregulation of all genes (Fig. 3f), with significant higher expression levels for those within Group-II (Fig. 3g). Together, these results highlight a set of overexpressed genes with hypermethylated CGIs in Alb-R26 $6^{\mathrm{Met}}$ tumours and identify a correlation between the location of hypermethylated CGIs and transcription status, where CGIs located further from the ATG showing predominantly increased transcription.

Next, we analysed whether Decitabine treatment would affect in vivo the expression levels of genes found hypermethylated and overexpressed in the $A l b-R 26^{\text {Met }}$ tumours. Focusing on a set of genes, we examined both their expression levels and the methylation levels of their corresponding CGIs in dissected tumours from $A l b-R 26^{M e t}$ mice either untreated or treated with Decitabine. RT-qPCR results showed that Decitabine treatment significantly decreases the expression levels of $7 / 8$ analysed genes (Fig. 4). Bisulfite sequencing studies revealed decreased methylation levels of most CpGs within the gene body CGIs (Fig. 4, Supplementary Data 5). Thus, CGI hypermethylation of genes belonging to Group-I and Group-II ensures their increased expression levels in $A l b-R 26^{M e t}$ tumours as demethylating treatment leads to a reduction of both CGI methylation content and transcription.

A CGI hypermethylation and gene overexpression signature defines a HCC patient subset. Next, we explored the relationship between changes in CGI methylation and gene expression in the above cohort of 41 HCC patients. Because of expected epigenomic heterogeneity between human samples, we reasoned it relevant to perform analyses in individual patients. We integrated transcriptome and methylome data to extract the expression levels of genes with differentially methylated CGIs (Fig. 5a), then classified patients according to the highest percentage of genes: (a) overexpressed with hypermethylated CpGs $\left(\mathrm{H}^{+} \mathrm{E}^{+}\right)$; (b) overexpressed with hypomethylated $\mathrm{CpGs}\left(\mathrm{H}^{-} \mathrm{E}^{+}\right)$; (c) underexpressed with hypermethylated $\mathrm{CpGs}\left(\mathrm{H}^{+} \mathrm{E}^{-}\right)$; (d) underexpressed with hypomethylated $\mathrm{CpGs}\left(\mathrm{H}^{-} \mathrm{E}^{-}\right)$. Intriguingly, 23/41 patients $(56 \%)$ showed an enrichment of genes overexpressed and with hypermethylated CpGs $\left(\mathrm{H}^{+} \mathrm{E}^{+}\right.$patient-subset; Fig. 5a, Supplementary Data 6), similar to the Alb-R26 $6^{M e t}$ model (Fig. 3b). Analysis of $M E T$ levels in HCC patients revealed that the mean MET levels in the $\mathrm{H}^{+} \mathrm{E}^{+}$subset is $0,77 \pm 0,16(9 / 23 ; 39 \%$ patients with $M E T$ levels $\geq 1)$, whereas in the " $\mathrm{NO} \mathrm{H}^{+} \mathrm{E}^{+}$" subset is $0,2 \pm 0,24(5 / 18$; $27 \%$ patients with $M E T$ levels $\geq 1$; Supplementary Data 6). Interestingly, all 7 patients belonging to the HCC subgroup-3 (in Fig. 1f) are characterised by more than $37 \%$ of genes both hypermethylated and overexpressed, and 5/7 patients belong to the $\mathrm{H}^{+} \mathrm{E}^{+}$subset (these 7 patients are highlighted with a red square and red \% in Fig. 5a). Next, we asked whether the $\mathrm{H}^{+} \mathrm{E}^{+}$patient subset could be also identified according to global gene expression or methylation features. Unsupervised cluster analysis of either global methylome or transcriptome data did not lead to the same patient clustering (Supplementary Fig. 7), thus strengthening the usefulness of combining methylation-expression features to identify specific HCC patient subsets.

The remarkable correlation between data obtained in the Alb$R 26^{\text {Met }}$ HCC model and analysed patient samples prompted us to perform integrative studies using another HCC model, for which methylation and expression data are available: the hepatitis- $B$ virus-X mice ( $H B x^{t g}$; GSE48052 27 . We first identified all CpGs differentially methylated in $H B x^{t g}$ HCC model, then correlated them with gene expression levels. We identified 115 genes both differentially methylated and differentially expressed (a very similar number to the 97 genes found in the Alb-R26 ${ }^{\text {Met }}$ genetic setting). Nevertheless, we found a different distribution compared to that of the Alb-R26 ${ }^{\mathrm{Met}} \mathrm{HCC}$, with an enrichment in genes both hypomethylated and downregulated (Supplementary Fig. 8). Next, we performed correlative analyses with the 41 HCC patients (reported in Fig. 5a): amongst the 18 " $\mathrm{NO} \mathrm{H}^{+} \mathrm{E}^{+}$" subset, 8 patients $(20 \%)$ share the same enrichment of hypomethylated and downregulated genes modelled by the $H B x^{t g}$ mice. Unexpectedly, only $1 / 8$ of these patients is reported positive for HBV. Thus, an epigenetic rewiring of gene sets through hypomethylation and downregulation occurs in a fraction of HCC patients, who do not appear to be characterised by the HBV-associated risk. Collectively, these findings indicate a rather intriguing specificity in how genes are epigenetically reprogrammed in HCC patients: an enrichment in hypermethylated and upregulated genes (for those corresponding to the Alb-R26 $6^{M e t}$ model) versus an enrichment in hypomethylated and downregulated genes (for those corresponding to the $H B x^{t g}$ model).

For the several genes found overexpressed and with hypermethylated CGIs in the $\mathrm{H}^{+} \mathrm{E}^{+}$patient subset, such as WT1, DLK1, TP73, EEF1A2, IGF1R, DKK1, SPOCK1, ITPKA, HOXA3, NOX4, FZD10, VASH2, GATA2, SOX8, their upregulation in HCC samples is coherent with their reported function as oncogenes in cancer. Concerning the $\mathrm{H}^{+} \mathrm{E}^{+}$patient subset, based on clinical data from TCGA, no association was found with a specific risk factor, such as $\mathrm{HBV} / \mathrm{HCV}$ infection, high-alcohol intake or non-alcoholic fatty liver disease (NAFLD) (Supplementary Fig. 9). Instead, the $\mathrm{H}^{+} \mathrm{E}^{+}$patient subset is distinguished by specific HCC molecular markers ${ }^{28}$. In particular, analysis of available RNA-seq data revealed a significant upregulation of alpha-FETOPROTEIN (AFP; a HCC marker when expressed in adult livers), JAG1, NOTCH3, NOTCH4, SOX9, VIM (progenitor markers) and CD24 (a HCC prognosis marker; Fig. 5b). Importantly, these markers are also upregulated in $A l b-R 26^{\mathrm{Met}}$ HCC (Fig. 5c), as we recently reported ${ }^{24}$. Together, these results show that an enrichment in genes characterised by "CGI hypermethylation and overexpression" occurs in HCC patients belonging to the so-called "proliferative-progenitor" subclass ${ }^{28}$. Moreover, these HCC patients share common features with the Alb-R26 ${ }^{\text {Met }}$ liver cancer model: the epigenetic $\mathrm{H}^{+} \mathrm{E}^{+}$signature and the "proliferative-progenitor" cell feature.

Overexpressed genes with hypermethylated CGIs in 5'-UTR or gene body regions act as oncogenes. The intriguing overlap between the Alb-R26 Met model and the $\mathrm{H}^{+} \mathrm{E}^{+}$patient subset prompted us to explore the relevance in cancer of the 55 genes found in $A l b-R 26^{\text {Met }}$ tumours both overexpressed and with hypermethylated CGIs either in the $5^{\prime}$-UTR or in the gene body region. For this analysis, transcriptome data from HCC patients were available for 51/55 genes (Supplementary Data 7). Remarkably, most genes are overexpressed in a large proportion of HCC patients (Fig. 6a, Supplementary Data 8), with a significant higher number in the $\mathrm{H}^{+} \mathrm{E}^{+}$patient subset compared with the other (Fig. 6b). These genes include PRRX1 (28 patients out of 41), CLDN7 (20), DBN1 (25), PCDH17 (30), PTK7 (21), ADAMTSL5 (21), ARHGAP21 (30), NFKB2 (23), CDKN2B (30), RELB (22), DUSP8 (24), SSBP4 (20), IRX3 (27), NEURL1B (19). Differences in the expression of these 51 genes permitted segregating the $\mathrm{H}^{+} \mathrm{E}^{+}$patient subset from the other (Fig. 6c). 

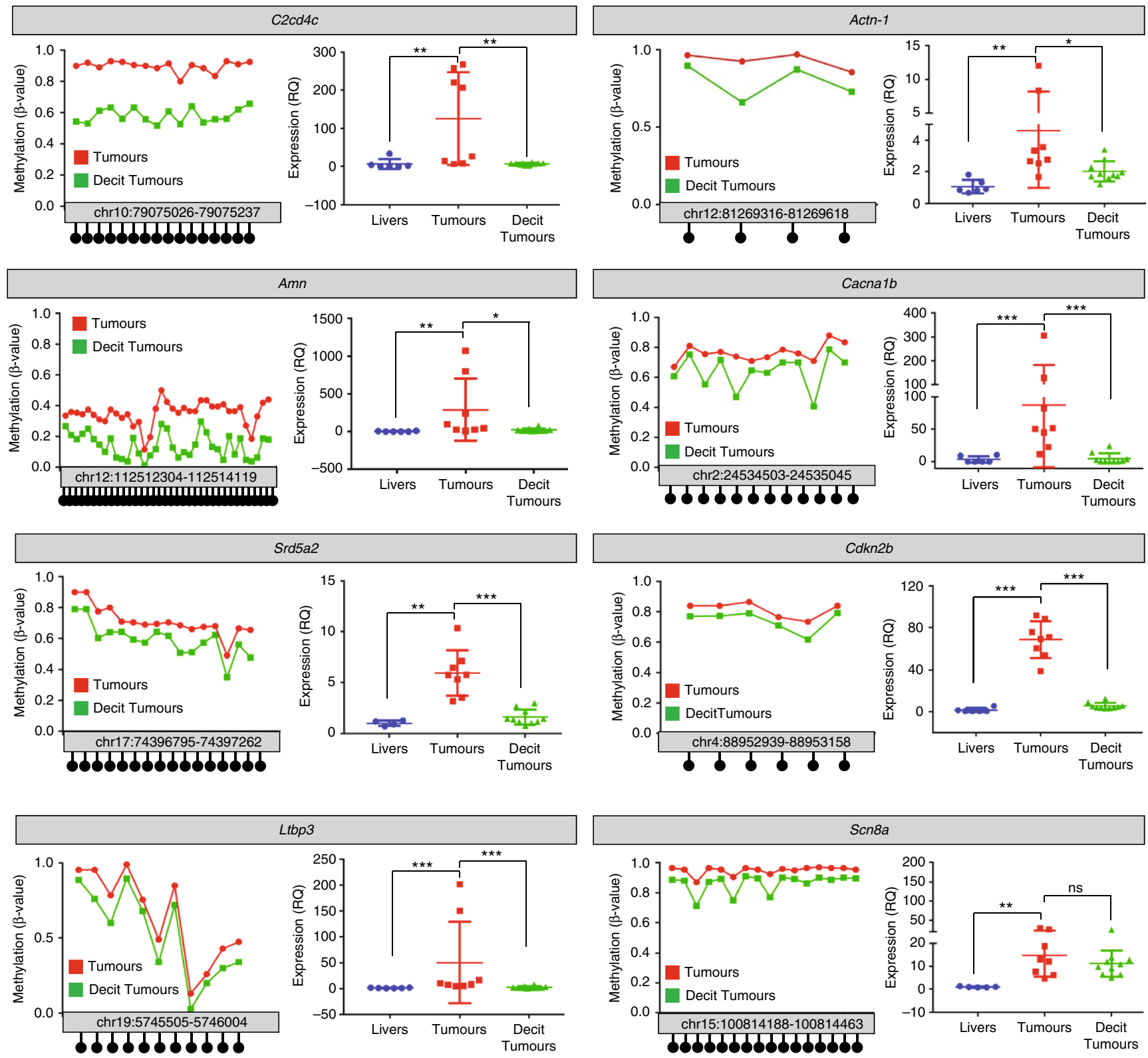

Fig. 4 Decitabine treatment decreases the expression and the CGI methylation levels of genes hypermethylated and overexpressed in Alb-R26Met tumours. Expression and CGI methylation levels of a set of genes found hypermethylated and overexpressed in the Alb-R26Met tumours were analysed in dissected tumours from Alb-R26 Met mice either untreated (red) or treated with Decitabine (green). For each indicated gene, graphs report the methylation levels of CpGs within the CGI of interest (left) and the expression levels of genes (right) in tumours from Alb-R26 Met mice either untreated (red) or treated with Decitabine (green), compared to control livers (blue). Note that demethylating treatment significantly decreased transcription levels. Concerning the Scn8a gene, the methylation levels of its gene body CGI was reduced in Decitabine treated tumours compared to untreated tumours. This was accompanied by a trend in downregulation of its expression levels, although not significant. It is possible that for $\mathrm{Scn} 8 \mathrm{a}$, the demethylation extent caused by the dose of Decitabine used is suboptimal to significantly influence its expression levels. Alternatively, a more complex mechanism could be involved in the regulation of Scn8a expression. Significance is indicated on the top. Not significant (ns): $P>0.05,{ }^{\star} P<0.05,{ }^{\star \star} P<0.01,{ }^{\star \star \star} P<0.001$

Furthermore, for each HCC patient we analysed the methylation levels of the CGIs corresponding to the 55 genes. We took into account that the number of CGIs for each gene varies between genes (Supplementary Data 7). 53/55 genes successfully liftedover from mouse to human, and both methylation and expression data are available for 51 genes. These analyses revealed that 42/51 $(82 \%)$ genes are both hypermethylated and overexpressed in at least 1 patient, and that $40 / 41(97,5 \%)$ patients have at least 1 gene both hypermethylated and overexpressed (Fig. 6d, e, Supplementary Fig. 10, Supplementary Data 9). Additionally, there is a significant higher number of genes both hypermethylated and overexpressed in the $\mathrm{H}^{+} \mathrm{E}^{+}$patient subset compared to the "NO $\mathrm{H}^{+} \mathrm{E}^{+"}$ subset $\left(\mathrm{H}^{+} \mathrm{E}^{+}\right.$versus "NO $\mathrm{H}^{+} \mathrm{E}^{+"}$ : $P$-value $<0.001$; Supplementary Fig. 10).

Curiously, in the $A l b-R 26^{M e t}$ cancer model $C d k n 2 a$, rather considered as a tumour suppressor, is overexpressed and hypermethylated in its gene body CGI, whereas no methylation changes were observed in its promoter CGI (Supplementary Data 4). We examined whether this phenomenon would also occur in HCC patients by analysing CDKN2A methylation and expression in HCC patients from TCGA and GSE56588 cohorts (for which methylation and expression data are available: 205/224 patients). 
a

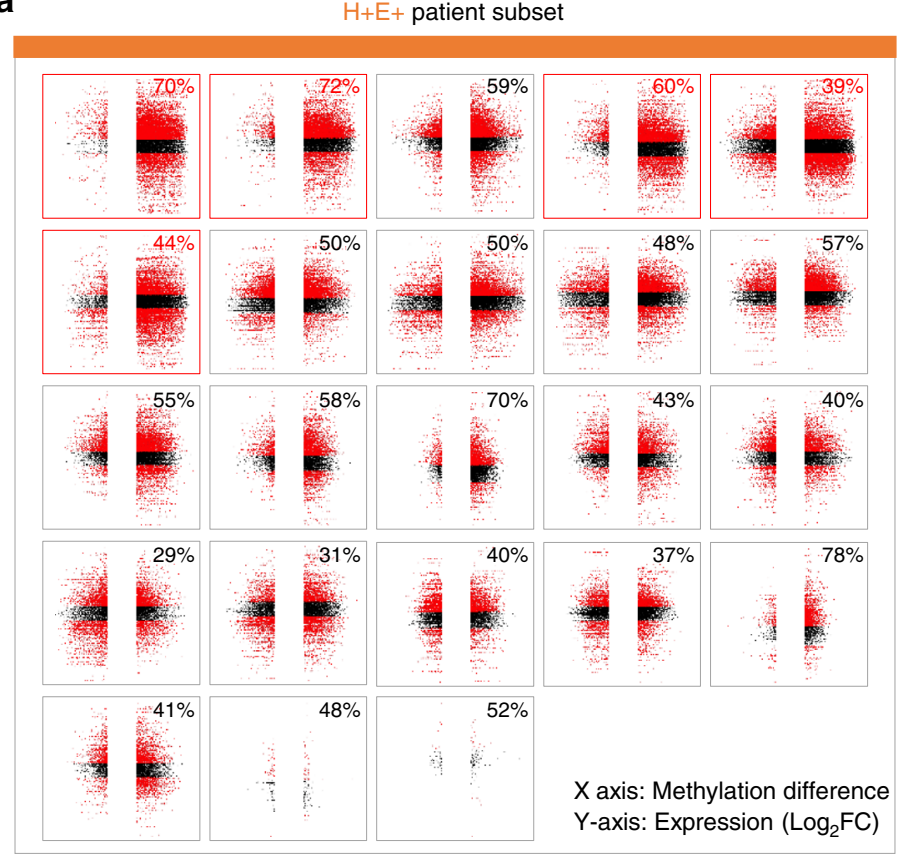

$\mathrm{NO} \mathrm{H}+\mathrm{E}+$ patient subset

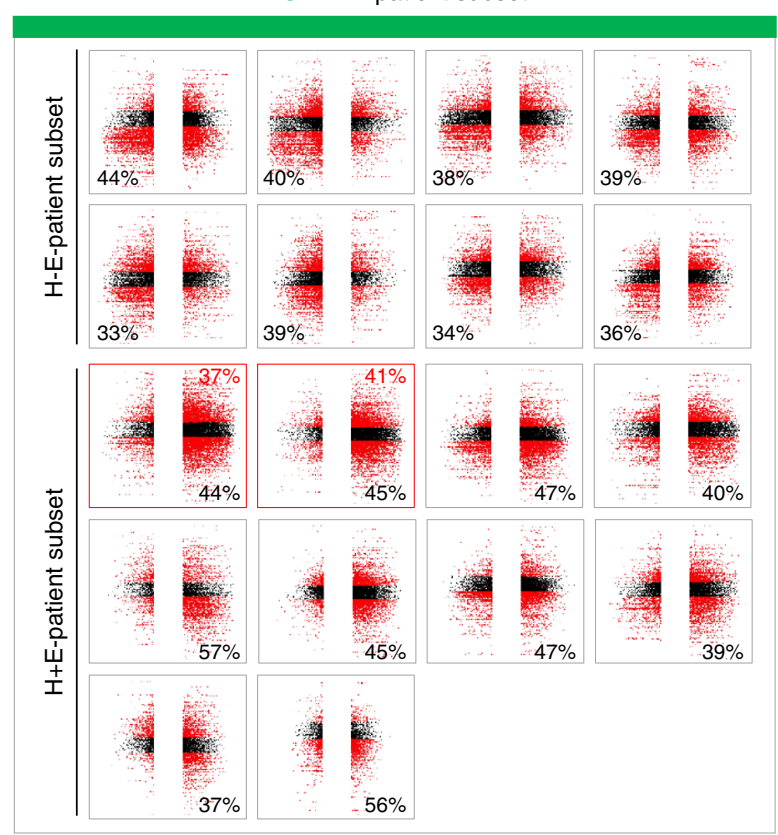

b
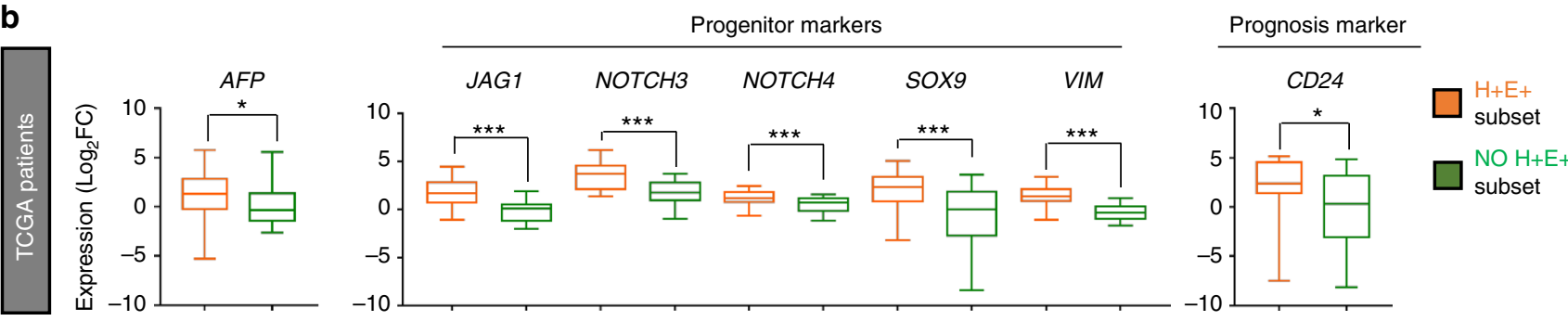

c
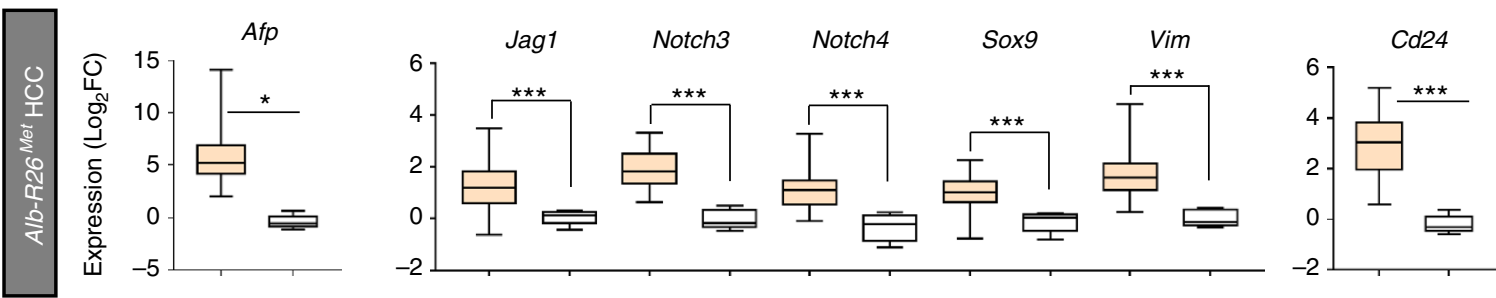

$\square$ AlbR26 ${ }_{\text {tumours }}^{\text {Met }}$

$\square$ Control

Fig. 5 A HCC patient subset, which is characterised by an enrichment of genes overexpressed and with hypermethylated CGls, belongs to the HCC "proliferative-progenitor" subclass. a The $41 \mathrm{HCC}$ patients are classified according to the highest percentage of genes over- versus underexpressed and with hyper- versus hypomethylated CGIs. In orange (left), patients with an enrichment of genes overexpressed and with $\mathrm{CGI}^{-}$hypermethylation ( $\mathrm{H}^{+} \mathrm{E}^{+}$ patient subset). Patients are organised according to the absolute number of hypermethylated CGIs. The percentage of genes overexpressed and hypermethylated is reported on the top. In green (right), all other patients are reported ( $\mathrm{NO} \mathrm{H}^{+} \mathrm{E}^{+}$patient subset). Note that this patient subset is characterised by an enrichment in downregulated genes. Patients are organised according to an enrichment of genes with CGI hypomethylation (top) and hypermethylation (bottom). Concerning the 7 patients of the HCC subgroup 3 identified in Fig. If (corresponding to the best overlap patients), 5 of them belong to the $\mathrm{H}^{+} \mathrm{E}^{+}$subset. Notably, all of these 7 patients are characterised by more than $37 \%$ of genes both hypermethylated and overexpressed (highlighted in panel with a red square and a red percentage of genes overexpressed with hypermethylated CGI). The $X$-axis reports methylation differences, whereas the $Y$-axis reports expression as $\log _{2} \mathrm{FC}$. b Transcript levels (from RNA-seq data) of the indicated genes in $\mathrm{H}^{+} \mathrm{E}^{+}$patient subset (in orange) versus the others (in green). Note significant high transcript levels of AFP, JAG1, NOTCH3, NOTCH4, SOX9, VIM and CD24 in the $\mathrm{H}^{+} \mathrm{E}^{+}$patient subset. c Transcript levels by RT-qPCR for the same genes shown in $\mathbf{b}$ analysed in Alb-R26 Met tumours versus control livers, displaying the same profile of gene upregulation as in the $\mathrm{H}^{+} \mathrm{E}^{+}$patient subset. Data have been reported in ref. ${ }^{24}$. Significance is indicated on the top. ${ }^{\star} P<0.05$, ${ }^{\star \star \star} P<0.001$

Mouse Cdkn2a has two CGIs: one in the promoter and another in the gene body. Instead, human CDKN2A has 5 CGIs: one in the promoter and four in the gene body. Data are available only for the CGI in the promoter and for one of the four CGIs located in gene body. Notably, in both cohorts we found an enrichment of patients with an overexpression of CDKN2A (39/41 and 166/204, in the respective cohorts), which is associated to a hypermethylation of the gene body CGI (21/39 and 163/166, in the respective cohorts). In contrast, not methylation changes have been detected in the promoter CGI for both HCC cohorts (Supplementary Fig. 11).

Analysing pathway enrichments in KEGG pathways of genes overexpressed with hypermethylated CGIs, we identified a significant enrichment of several cancer-related pathways, such 
a

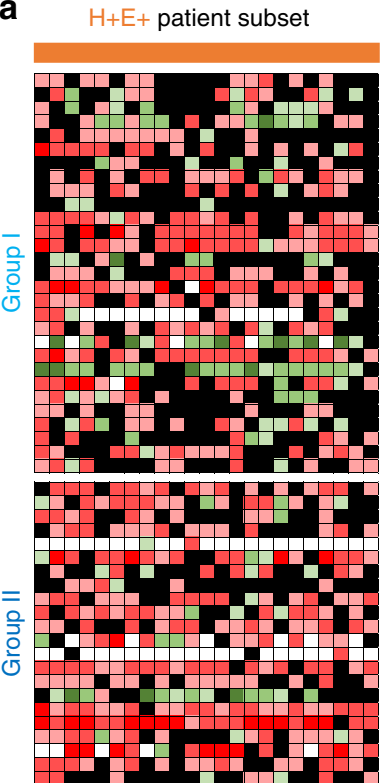

$\mathrm{NO} \mathrm{H}+\mathrm{E}+$ patient subset

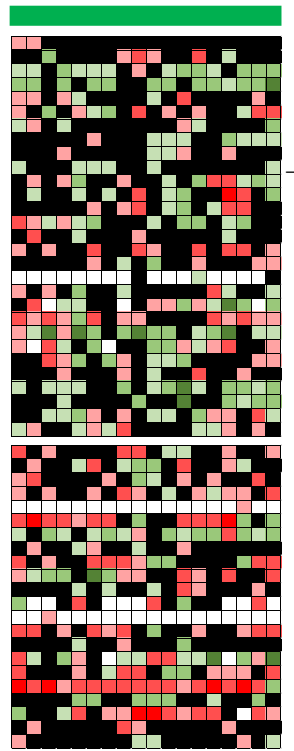

d

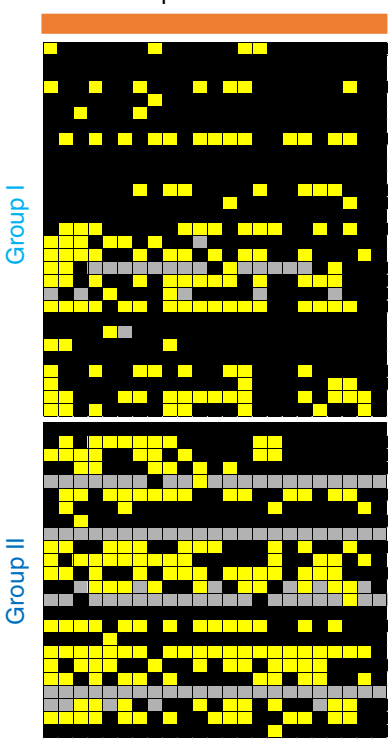

$\mathrm{NO} \mathrm{H}+\mathrm{E}+$ patient subset

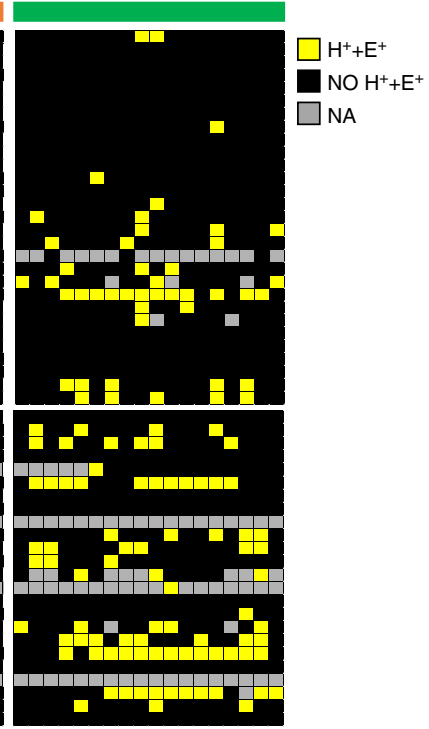

b

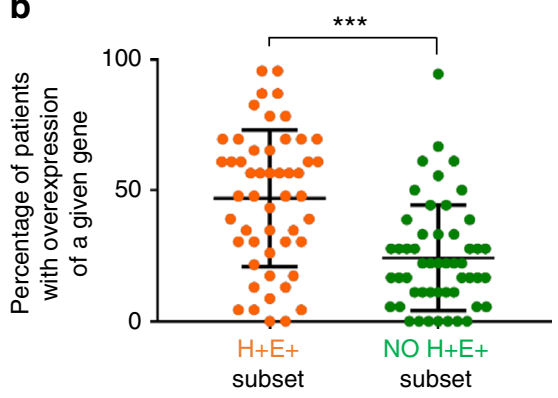

C

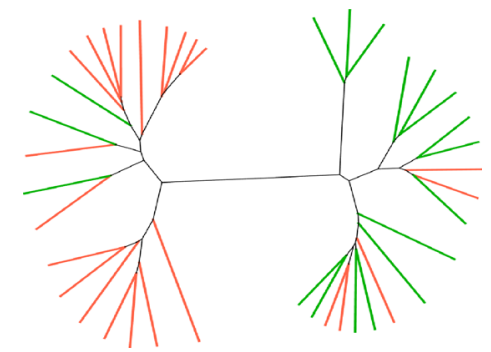

e

\begin{tabular}{|c|c|c|c|}
\hline$N^{\circ}$ genes $\mathrm{H}^{+} \mathrm{E}^{+}$ & $\mathrm{N}^{\circ}$ patients & $\mathrm{N}^{\circ}$ patients & $\mathrm{N}^{\circ}$ genes $\mathrm{H}^{+} \mathrm{E}^{+}$ \\
\hline $42(82 \%)$ & A least 1 & $40(97 \%)$ & At least 1 \\
\hline $28(55 \%)$ & A least 5 & $36(88 \%)$ & At least 5 \\
\hline $20(39 \%)$ & At least 10 \\
\hline $4(8 \%)$ & At least 20 & $23(56 \%)$ & At least 10 \\
\hline $3(7 \%)$ & At least 20 \\
\hline
\end{tabular}

f

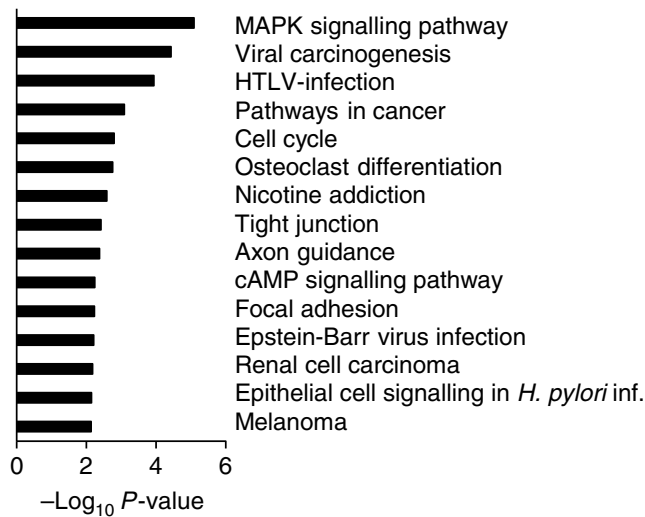

Fig. 6 The 55 genes identified in Alb-R26Met tumours are also upregulated in the $\mathrm{H}^{+} \mathrm{E}^{+}$patient subset, and a large proportion of them is characterised by hypermethylated CGIs. a Heat-map reporting expression levels of genes found overexpressed and with hypermethylated CGls in Alb-R26 Met tumours versus control livers (rows; subdivided in Group I and II and organised according to the relative position to the ATG) in individual HCC patients (columns; organised as in Fig. 5a). Red: upregulated genes; green: downregulated genes. Black: not differentially expressed. White: data not available. The scale is shown on the right (expressed as $\log _{2} \mathrm{FC}$ ). The $\mathrm{H}^{+} \mathrm{E}^{+}$patient subset is highlighted in orange (left), whereas all other patients in green (right). $\mathbf{b}$ In the graph, each dot corresponds to a given gene (the total 51 genes are reported). Their position corresponds to the percentage of patients in which the gene is overexpressed. In orange (left), for the $\mathrm{H}^{+} \mathrm{E}^{+}$patient subset. In green (right), for all other patients. c Unrooted distance tree of $\mathrm{HCC}$ patients based on the 51 genes identified in the Alb-R26 Met tumours. In orange: $\mathrm{H}^{+} \mathrm{E}^{+}$patient subset. In green: all other patients. d Heat-map highlighting with a yellow square genes overexpressed and with hypermethylated CGI in the corresponding HCC patient. The heat-map is organised as in panel A. Black square: genes not simultaneously overexpressed and with hypermethylated CGI in the corresponding HCC patient. Grey: data not available. e Tables reporting the numbers with percentages of genes overexpressed and with hypermethylated CGI in at least 1, 5, 10 or $20 \mathrm{HCC}$ patients (left), as well as numbers with percentages of patients with at least 1, 5, 10 or 20 genes overexpressed and with hypermethylated CGI out of the 55 genes identified in the Alb-R26Met model (right). f Histogram reporting the KEGG pathway enrichment analysis for the 55 genes identified in Alb-R26 Met tumours, ordered according to the - Log ${ }_{10} P$-value. Significance is indicated on the top. ${ }^{\star \star \star} P<0.001$

as MAPK signalling, viral carcinogenesis, pathways in cancer, cell cycle (Fig. 6f, Supplementary Fig. 12). Consistently, some of these genes are well-known oncogenes, such as GRB10, MAP3K6, JUN (which belong to MAPK pathway), NFKB2, RELB (which belong to NFkB and MAPK pathways), MET, PTK7 (Supplementary
Data 10). The presence of poorly characterised genes among wellestablished oncogenes prompted us to explore their functional relevance in cell tumorigenic properties. Focusing on Scn8a, Actn1, Srd5a, NFkB2 and Neurl1b, we used shRNA-mediated targeting to lower their expression levels in Alb-R26 ${ }^{\text {Met }} \mathrm{HCC}$ cells 
(Fig. 7a, Supplementary Fig. 13). Stable clones were used to assess cell tumorigenic properties in vitro and in vivo. These genes were selected because: (1) of their overexpression in HCC patients (SCN8A in $41 \%, A C T N 1$ in $22 \%$, SRD $5 A 2$ in $5 \%, N F k B 2$ in $56 \%$, NEURL1B in $46 \%$ ); (2) of their action as oncogenes in cancer cells (and particularly in HCC) has been less explored in previous studies (with the exception of NFkB2 and SRD5A). Downregulation of either $S c n 8 a, A c t n 1, S r d 5 a, N F k B 2$ or Neurl1b interferes with the capability of cells to form: (a) colonies in anchorage-independent assays (Fig. 7b); (b) foci in anchoragedependent assays, as revealed by a significant smaller foci size even if numbers were similar (Fig. 7c); (c) tumour spheres when cells were grown in self-renewal conditions (Fig. 7d); (d) tumours in nude mice xenografts (Fig. 7e). Collectively, these data show that most of the 55 genes identified in the $A l b-R 26^{\text {Met }}$ cancer model are also overexpressed and with hypermethylated CGIs in a large proportion of HCC patients, with a set of them acting together as an "oncogenic module".

\section{Discussion}

The increasing knowledge on how epigenetic modifications such as DNA methylation influence patterns of gene expression in cancer holds great promises for understanding biological processes, as well as for their use in prognosis, patient stratifications and therapeutic intervention ${ }^{3,29}$. This is well exemplified by reports showing correlations between changes in CGI methylation and a remarkable resetting of transcriptional networks in cancer. In the present study, we employed a clinically relevant cancer mouse model in which tumorigenesis is triggered by a slight perturbation in signalling dosages rather than drastic genetic modifications, to examine the DNA methylation landscape associated with tumorigenic acquisition. We reasoned that such a genetic tool offers a unique way to model DNA methylation changes occurring in human cancerogenesis in the absence of drastic alterations of epigenetic modulators. Our genome-wide strategy highlighted key correlations between site-specific DNA methylation changes and transcriptional dosages of the corresponding genes. The type of changes found for some genes belong to the well-known mechanism of downregulation of tumour suppressors through promoter DNA hypermethylation, which was the case of Oat and Igfbp5 that can act as tumour suppressors in certain cellular contexts. Quite unexpectedly, however, there is an enrichment of genes both overexpressed and with hypermethylated CGIs. Several of them are well-known oncogenes, such as Grb10, Map3k6, Jun, RelB, Met, Ptk7, as well as NF-KB2, $\operatorname{Srd5a2}$, which have been functionally validated in this study together with others poorly investigated so far: Scn8a, Actn1, Neurl1b. Results from our functional assays in Alb-R26 ${ }^{\text {Met }}$ HCC cells demonstrate how downregulating each individual oncogene reduces, but not abolishes, cell tumorigenic properties. These results conceptually illustrate that, although each oncogene contributes to the tumorigenic properties of cancer cells, they operate in a cooperative manner as an "oncogenic module" for ensuring robustness of the tumorigenic program.

Our integrative studies using human HCC databases demonstrate that enrichment in genes both overexpressed and with hypermethylated CGIs also characterises $56 \%$ of the HCC patients, which we named as the " $\mathrm{H}^{+} \mathrm{E}^{+}$patient subset". For several genes, upregulation in expression levels is coherent with them being bona fide oncogenes. For example, it is the case of WT1, DLK1, TP73, EEF1A2, IGF1R, DKK1, SPOCK1, ITPKA, HOXA3, NOX4, FZD10, VASH2, GATA2, SOX8. Thus, our genetic studies together with a revisited analysis of human cancer databases reveal that raising dosages of oncogene sets characterised by hypermethylated CGIs is a robust mechanism operating in cancer. The existence of such events in human pathology supports the clinical relevance of these findings. Remarkably, the $\mathrm{H}^{+} \mathrm{E}^{+}$patient subset belongs to the "HCC proliferative-progenitor" subclass, thus attributing an additional feature to this aggressive HCC subtype. For clinical implementation, integrative methylome and transcriptome analyses on additional $\mathrm{HCC}$ cohorts will demonstrate the robustness of $\mathrm{H}^{+} \mathrm{E}^{+}$ patient subset classification. These findings also raise the question as to whether the $\mathrm{H}^{+} \mathrm{E}^{+}$patient subset could be most sensitive to therapies based on demethylation agents ${ }^{30}$.

Our expression analyses revealed that $\mathrm{H}^{+} \mathrm{E}^{+}$genes can be segregated into two groups, according to their relative position to the ATG, with overall significant higher expression levels observed the further the CGI is located from the ATG (Group-II). Whereas for Group-I the relative position of the hypermethylated CGIs falls predominantly with the $5^{\prime}$-UTR, their location in Group-II is in the gene body. The positive correlation between gene body hypermethylation and expression is coherent with previous studies based on in vitro modulation of the methylation content in cancer cell lines ${ }^{12}$. Additionally, single-base resolution DNA methylation profiling combined with transcriptome analysis correlated changes in gene expression levels with the $\mathrm{CpG}$ methylation content in gene body ${ }^{31-34}$. The significance of gene body DNA methylation on transcriptional regulation is strengthened by studies exploring correlations with chromatin modifications. It has been reported that in the gene body: (a) $\mathrm{H} 3 \mathrm{~K} 4 \mathrm{me} 3$ association to alternative promoters depends on their CpG methylation content, impacting alternative transcript products $^{35}$; (b) H3K36me3 associates with methylated DNA in gene body and permits transcription ${ }^{36}$; (c) CTCF binding is lost in hypermethylated CGI, influencing splicing, in addition to the well-known action of CTCF in maintenance of chromatin architecture through generation of chromatin barriers ${ }^{37,38}$; (d) $\mathrm{H} 3 \mathrm{~K} 27 \mathrm{me} 3$ and $\mathrm{H} 3 \mathrm{~K} 9 \mathrm{me} 3$, known as repressive histone marks, are not associated with methylated $\mathrm{DNA}^{39}$. Future studies integrating methylome, transcriptome and ChIP-seq with several chromatin marks like those mentioned above will contribute to uncover the underlying mechanisms of action of oncogene upregulation through gene body methylation. Taking into account the variety of chromatin factors found associated in gene body, it is likely that different sets of genes are modulated by different mechanisms of action.

For translating these findings into therapies, an intriguing question is whether and to what extent the epigenetic reprogramming of a set of genes acting as an "oncogene module" still leaves space for tumour vulnerability. Our functional studies show that targeting each individual oncogene reduces, but not abolishes, tumorigenicity, indicating that each oncogene provides a net contribution to the whole tumorigenic properties of cancer cells. Such context may be particularly relevant for tumours that are not predominantly "addicted" to genetic mutation $(\mathrm{s})^{40}$, such as HCC. This likely explains the partial response of HCC patients even with most promising drugs targeting one or at least a restricted number of targets (e.g., Sorafenib). Such scenario contrasts with exceptional cases of effectiveness, due to the stringent addiction of cancer cells to a given oncogene, such as BCR-ABL in chronic myeloid leukaemia, ERBB2 in breast cancer, ERBB1 in non-small cell lung cancer, B-RAF in metastatic melanoma. To identify vulnerability, an approach could be to extract enriched pathways that are deregulated from the whole list of epigenetically reprogramed genes. In the case of tumorigenesis modelled by the Alb-R26 $6^{M e t}$ mice, the MAPK signalling cascade is on the top of the list of enriched pathways (with 11 genes differentially methylated in tumour versus control livers). Through a phosphokinome-based educated guess drug screen, we recently reported that tumorigenesis modelled by the $A l b-R 26^{M e t}$ genetic 


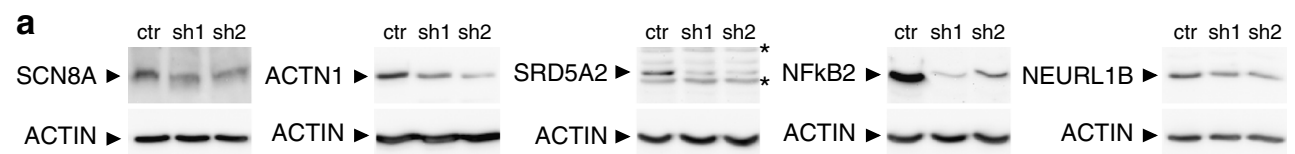

b Anchorage-independent growth

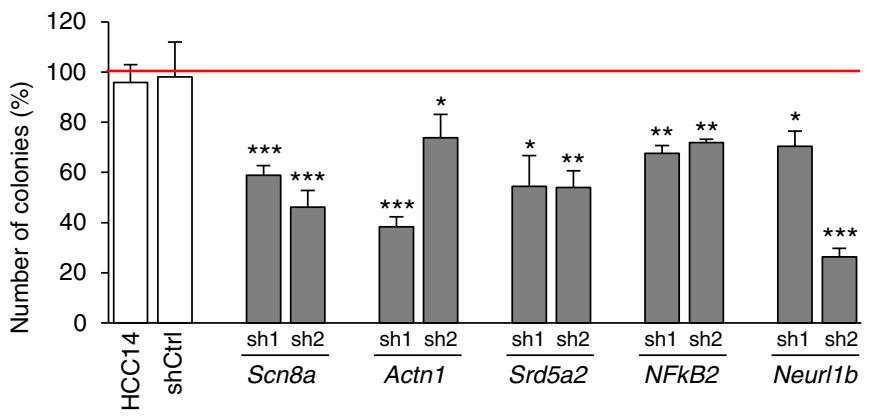

C

Anchorage-dependent growth
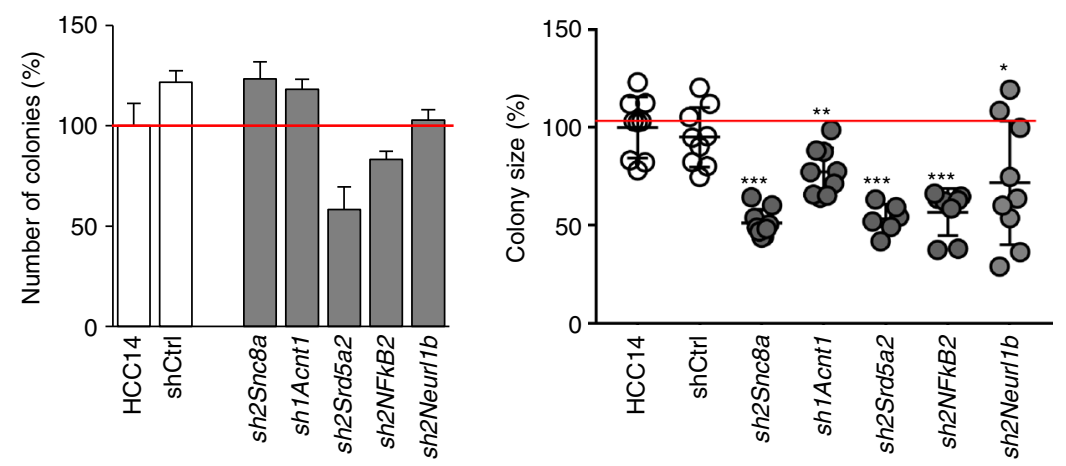

d

e

Xenograft
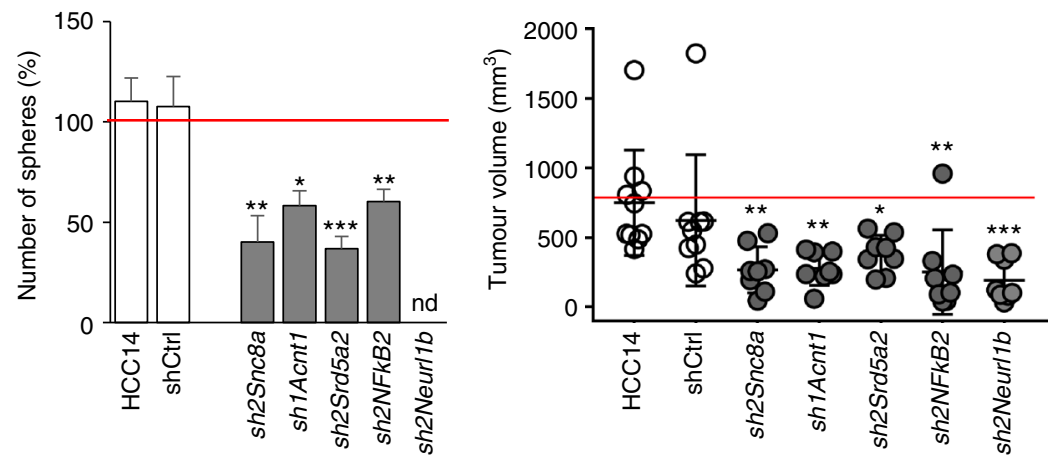

Fig. 7 Downregulation of overexpressed genes with hypermethylated gene body CGI in Alb-R26 Met HCC cells interferes with their tumorigenic properties both in vitro and in vivo. a Western blots showing SCN8A, ACTN1, SRD5A2, NFkB2 and NEURL1B protein levels in stable clones established after transfection of $A l b-R 26^{\text {Met }} \mathrm{HCC14}$ cells with plasmids carrying a shRNA sequence targeting the corresponding gene. Protein levels were compared to control cells (ctr). ACTIN was used as a loading control in all western blots. The asterisk indicates nonspecific bands detected using anti-SRD5A2 antibodies. b-e Biological assays to assess functional properties of Alb-R26 Met $\mathrm{HCC} 14$ cells carrying a shRNA sequence targeting candidate genes. Effects were compared to $\mathrm{HCC14}$ cells either untransfected or transfected with a control shRNA (shCtrl). b Graph reporting the number of colonies formed in anchorage-independent growth assays using 2 different shRNA targeting sequences for each candidate gene. Note a decrease in colony number formation of cells with downregulated candidate genes compared with control cells. c Graphs reporting the number (left) and the size (right) of colonies formed in anchorage-dependent growth assays. Whereas no significant changes in colony numbers were detected, note a significant decrease in colony size when the candidate gene is downregulated. $\mathbf{d}$ Graph reporting number of spheres formed in tumour sphere assays. Note that downregulation of candidate genes significantly reduces sphere number formation. e Graph reporting the tumour volume of mice injected either with Alb-R26 ${ }^{\mathrm{Met}} \mathrm{HCC} 14$ control cells or with Alb-R26 Met $\mathrm{HCC14}$ cells carrying a shRNA sequence targeting candidate genes. Note that downregulation of candidate genes significantly interferes with the in vivo tumorigenic properties of $A l b-R 26^{M e t} \mathrm{HCC14}$ cells. Significant differences between groups are indicated on the top. ${ }^{\star} P<0.05,{ }^{\star \star} P<0.01,{ }^{\star \star \star} P<$ 0.001 (nd: no determined) 
system is vulnerable to Ras pathway targeting, provided that its inhibition occurs concomitantly while destabilising the stress support mitochondrial pathway ${ }^{24}$. It is therefore tempting to speculate that a proportion of tumours, particularly those with epigenetic reprogramming rather than those with drastic genomic instability, still maintains vulnerability to synthetic lethal interactions. Alternatively, the use of epigenetic modulating agents to reprogram a set of genes in cancer cells, ideally used at minimal doses to limit side effects ${ }^{3,29}$, could reinforce the action of promising targeted therapies that, when used alone, have been unsatisfactory in clinical trials, as reported for chronic myeloid leukaemia ${ }^{41}$. We show here that Decitabine treatment reduces the methylation levels of gene body CGIs and the expression levels of the corresponding genes. Such event correlates with reduced tumorigenic properties of Alb-R26 ${ }^{\text {Met }}$ HCC cells. Nevertheless, cells for 10 days in culture after $48 \mathrm{~h}$ with Decitabine recover their tumorigenic properties (Supplementary Fig. 14), illustrating how reduced tumorigenicity by transient demethylation treatment is reversible, likely by resetting increased levels of oncogenes. This would be coherent with previous studies showing the capability of cancer cells to restore acquired epigenetic modifications ${ }^{12}$. Collectively, these findings support the possibility of achieving effective response in cancer combining epigenetic modulating agents with targeted treatments.

In conclusion, by exploring epigenetic changes associated with tumorigenesis in a clinically relevant mouse model, we discovered that for oncogene sets, characterised by hypermethylated CGIs either in their $5^{\prime}$-UTR or in the gene body, their expression levels are raised in cancer. The use of a mouse model in which tumorigenesis is not caused by drastic genetic manipulations strengthens the advantage of disrupting multiple oncogenes through an epigenetic reprogramming. Delineating the relationship between aberrant DNA methylation and expression of oncogenes/tumour suppressors will likely contribute to identify biomarkers for patient stratifications, functional pathways operating in cancer and strategies for an epigenetic restoration of deregulated genes in combination with molecular therapies.

\section{Methods}

Mice. Ethics Statement: All procedures involving the use of animals were performed in accordance with the European Community Council Directive of 22 September 2010 on the protection of animals used for experimental purposes (2010/63/UE). The experimental protocols were carried out in compliance with institutional Ethical Committee guidelines for animal research (comité d'éthique pour l'expérimentation animale-Comité d'éthique de Marseille; agreement number D13-055-21 by the Direction départementale des services vétérinairesPréfecture des Bouches du Rhône).

Alb-R26 $6^{\text {Met }}$ mice: $R 26^{\text {stopMet }}$ and Alb-R26 $6^{M e t}$ mice have been described previously ${ }^{42,43}$. Briefly, R26 $6^{\text {stopMet }}$ mice (international nomenclature Gt(ROSA) 26Sortm1 (Actb-Met)Fmai) carrying a conditional mouse-human chimeric Met transgene in the Rosa26 locus were crossed with Albumin-Cre mice (B6.Cg-Tg(Albcre) $21 \mathrm{Mgn} / J$ ) obtained from the Jackson Laboratory. All mice were maintained on a $50 \%$ mixed 129/SV and C57BL6 background. Mice were genotyped via PCR analysis of genomic DNA as reported in previous studies ${ }^{42,43}$. Mice were housed under pathogen-free conditions.

Mice drug treatment: For in vivo demethylation experiments to asses methylation levels of selected CGIs, as well as expression levels of the corresponding genes, Alb-R26 ${ }^{\mathrm{Met}}$ mice were treated with intraperitoneal injections of $2.5 \mathrm{mg} / \mathrm{kg}$ of Decitabine, twice per week (for a total of three injections).

DNA/RNA-related experiments. Genomic DNA isolation: Genomic DNA from $A l b-R 26^{M e t}$ tumours and control livers was prepared using the ZR Genomic DNA Tissue Miniprep (Zymo Research Company), according to the manufacturer's instructions.

Total RNA extraction: Total RNA from frozen tissues and cultured cells was isolated using the RNeasy Mini Kit (Qiagen), according to the manufacturer's instructions. DNase (Qiagen) treatment was included to avoid possible genomic DNA contamination. Regarding frozen samples, $20 \mathrm{mg}$ of tissue were first homogenised in the specific lysis buffer by 6300 r.p.m. $2 \times 30$ s using Precellys 24 (Bertin technologies), then the RNeasy Mini Kit (Qiagen) was used.
cDNA and quantitative RT-PCR analysis: cDNA was synthesised using a Reverse Transcription Kit (Bio-Rad). PCR reactions were performed using 2X SYBR Green qPCR SuperMix-UDG with Rox (ThermoFisher Scientific) and specific primers $(1 \mu \mathrm{M}$; qPCR primer sequences are listed in Supplementary Data S11). Expression levels were quantified using the comparative Ct method $\left(2^{-\Delta \Delta C T}\right.$ method) with the house-keeping gene Hprt as a control for internal normalisation, and results are expressed as $\mathrm{RQ}=2^{-\Delta \Delta \mathrm{CT}}$.

High-throughput sequencing. Comparative Genomic Hybridisation analysis: Genomic DNA form dissected Alb-R26 ${ }^{\text {Met }}$ tumours $(n=16)$ and control livers $(n=8)$ was analysed by the "Plateforme Biopuces et Sequencage IGBMC" (Illkirch, France) using an Agilent Oligonucleotide Array-Based CGH for Genomic DNA Analysis (CGH microarray $4 \times 180 \mathrm{~K}$ ).

Genome-wide DNA methylation analysis: Methyl-MiniSeq EpiQuest genomewide sequencing was perform using genomic DNA from dissected Alb-R26 ${ }^{\mathrm{Met}}$ tumours $(n=10)$ and control livers $(n=3)$ to analyse the DNA methylation profile by the Zymo Research Corporation (Irvine, CA, USA).

Library construction. Libraries were prepared from 200-500 ng of genomic DNA digested with 60 units of TaqaI and 30 units of MspI (NEB) sequentially, then extracted with Zymo Research DNA Clean and Concentrator ${ }^{\text {ris }}-5$ kit (Cat\#: D4003). Fragments were ligated to pre-annealed adapters containing 5 '-methylcytosine instead of cytosine according to Illumina's specified guidelines (www. illumina.com). Adaptor-ligated fragments of $150-250 \mathrm{bp}$ and $250-350 \mathrm{bp}$ in size were recovered from a $2.5 \%$ NuSieve 1:1 agarose gel (Zymoclean ${ }^{\text {tx }}$ Gel DNA Recovery Kit, Zymo Research Cat\#: D4001). The fragments were then bisulfite treated using the EZ DNA Methylation-Lightning ${ }^{\mathrm{Tm}}$ Kit (Zymo Research, Cat\#: D5020). Preparative-scale PCR was performed and the resulting products were

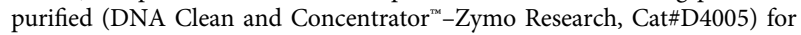
sequencing on an Illumina HiSeq.

Alignments and data analysis. Sequence reads from bisulfite-treated EpiQuest libraries were identified using standard Illumina base-calling software and then analysed using a Zymo Research proprietary analysis pipeline, which is written in Python and used Bismark (http://www.bioinformatics.babraham.ac.uk/projects/ bismark/) to perform the alignment. Index files were constructed using the Bismark-genome-preparation command and the entire reference genome. The non-directional parameter was applied while running Bismark. All other parameters were set to default. Filled-in nucleotides were trimmed off when doing methylation calling. The methylation level of each sampled cytosine was estimated as the number of reads reporting a $\mathrm{C}$, divided by the total number of reads reporting a $\mathrm{C}$ or $\mathrm{T}$ ( $\beta$-value).

Overall sequencing results (for 13 samples) are: (a) mean total read: 30 million read pairs, (b) mean mapping efficiency: $40 \%$, (c) mean unique CpGs: 4.1 millions, (d) mean average CpG coverage: $16 \times$, (e) mean bisulfite conversion rate: $98 \%$. Data accessibility: Methylome datasets generated from this study are deposited with the Gene Expression Omnibus (accession GSE90093).

Identification of differentially methylated CpGs. A total of 1.085 .757 unique single $\mathrm{CpG}$ sites, present in all samples, were analysed. $\beta$-value ranged from 0 (not methylated) to 1 (fully methylated). To identify differentially methylated CpGs, the methylation difference per CpG was calculated as the mean $\beta$-value of tumours minus the mean $\beta$-value of controls. Those with a methylation difference $>0.2$ were filtered to retain the ones with a FDR $<0.05$ (Student's two-sided $T$-test and Benjamini-Hochberg False Discovery Rate for $P$-value correction). A CpG is classified as "hypomethylated" when the methylation difference is $<-0.2$ and as "hypermethylated" when the methylation difference is $>0.2$. A global analysis was first carried out with all measured CpGs, then dividing the CpGs according to their location within or outside a CGI (CpG Island bedfile downloaded from UCSC). According to the Methyl-MiniSeq EpiQuest coverage, the CGI coverage by CpGs was $87.5 \%$. Studies were focused on CGI regions. The overlap with CGI and the annotated gene was performed using the CGI track from the UCSC genome browser, and Refseq gene annotations based on the NCBI37/mm 9 mouse reference. We discarded "ubiquitous CpGs" located in more than one annotated gene, and we extended the gene/CGI annotation to the gene's promoter region to $-1.5 \mathrm{~kb}$ upstream the TSS.

Targeted Bisulfite Sequencing: Genomic DNA from Alb-R26 ${ }^{\text {Met }}$ tumours dissected from mice treated with Decitabine $(2.5 \mathrm{mg} / \mathrm{kg}$; twice per week, for a total of three treatments; $n=4)$ and without treatment $(n=2)$ was used to asses CpG methylation levels in selected regions within the candidate CGIs through bisulfite sequencing by the Zymo Research Corporation (Irvine, CA, USA).

Assay Design, Sample Preparation and Multiplex Targeted Amplification. After assessment of DNA concentration and quality, DNA samples were bisulfite converted using the EZ DNA Methylation-LightningTM Kit (ref Cat\#D5030) according to the manufacturer's instructions. Primers were designed with Rosefinch, Zymo Research's proprietary sodium bisulfite converted DNA-specific primer design tool (primer sequences are listed in Supplementary Data 5). Multiplex amplification of all samples using the specific primer pairs and the Fluidigm Access ArrayTM System was performed according to the manufacturer's instructions. The resulting amplicons were pooled for harvesting and subsequent barcoding according to the Fluidigm instrument's guidelines. After barcoding, samples were purified (ZR-96 DNA Clean and Concentrator ${ }^{\mathrm{TN}}-\mathrm{ZR}$, Cat\#D4023), then prepared for parallel sequencing using a MiSeq V2 $300 \mathrm{bp}$ Reagent Kit and paired-end sequencing protocol, according to the manufacturer's guidelines. 
Targeted Sequence Alignments and Data Analysis. Sequence reads were identified using standard Illumina base-calling software and then analysed using a Zymo Research proprietary analysis pipeline, which is written in Python. Sequence reads were aligned back to the reference genome using Bismark (http://www. bioinformatics.babraham.ac.uk/projects/bismark/), an aligner optimised for bisulfite sequence data and methylation calling ${ }^{44}$. Paired-end alignment was used as default thus requiring both read 1 and read 2 be aligned within a certain distance, otherwise both read 1 and read 2 were discarded. Index files were constructed using the bismark_genome_preparation command and the entire reference genome. The non-directional parameter was applied while running Bismark. All other parameters were set to default. The methylation level of each sampled cytosine was estimated as the number of reads reporting a $\mathrm{C}$, divided by the total number of reads reporting a $\mathrm{C}$ or $\mathrm{T}$.

Transcriptome analysis by RNA-seq: Total RNA from dissected Alb-R26 ${ }^{\text {Met }}$ tumours $(n=4)$ and control livers $(n=4)$ was processed for transcriptome analysis. RNA quality was controlled using the Agilent RNA 6000 Pico Kit and Agilent 2100 Bioanalyzer (Agilent Technologies, Santa Clara, California) according to the manufacturer's recommendations. Total RNA ( $1 \mu \mathrm{g}$ per sample) was used for library preparation using the TruSeq RNA Sample Preparation Kit (Illumina) by GATC Biotech (Mulhouse; NGSelect service). Sequencing was performed on a HiSeq 2500 (Illumina; $2 \times 50$ bp paired end) and base calling performed using RTA (Illumina). Quality control of raw reads was done using FastQC (http://www. bioinformatics.babraham.ac.uk/projects/fastqc/). Reads were mapped to the reference genome $\mathrm{mm} 9$ with STAR aligner ${ }^{45}$ using default parameters; differential expression was calculated using the Cufflinks package ${ }^{46}$.

Cell culture-related experiments. Cell lines: $A l b-R 26^{\text {Met }} \mathrm{HCC}$ cell lines (HCC3, HCC13 and HCC14) were established, characterised and cultured as previously described ${ }^{24}$; cells were regularly tested by PCR-based assay to confirm their maintenance in free Mycoplasma culture condition.

shRNA-mediated downregulation of candidate genes: The functional relevance of candidate oncogenes was determined using shRNA targeting sequences (Sigma; shRNA sequences are reported in Supplementary Data 12). In particular, plasmids carrying the shRNA sequence were transfected in cells using Lipofectamine 2000 reagent, according to the manufacturer' instructions (ThermoFisher Scientific). After 1 week of puromycin selection, pools of resistant clones were used to verify downregulation of gene expression levels (by RT-qPCR and western blots) and to perform biological assays.

Cell drug treatment: For the demethylation experiments, cells were exposed to $0.3 \mu \mathrm{M}$ of Decitabine (5-Aza-2'-deoxycytidine; Selleckchem) for $48 \mathrm{~h}$. After treatment, cells were used for cell viability, anchorage-dependent growth assay, anchorage-independent growth assay, tumour sphere formation assay and xenograft studies. For experiments shown in Supplementary Fig. 14, after $48 \mathrm{~h}$ of Decitabine treatment, cells were cultured for 10 days with complete media before performing the experiments.

Survival assay: Cells were seeded in a $150-\mu \mathrm{l}$ volume per well in 96 well plates $(10,000$ cells/well $)$ in $10 \%$ serum for $24 \mathrm{~h}$, then Decitabine treatment was applied at $0.3 \mu \mathrm{M}$ in the corresponding wells. After $48 \mathrm{~h}$, cell viability was assessed in a Cell Titer Glo Luminescent Assay (Promega) and luminescent signals were measured with a luminometer microplate reader (Berthold). Data are expressed as means \pm SEM of three independent experiments performed in triplicate.

Anchorage-dependent growth assay (focus formation assay): To measure anchorage-dependent growth, 300 cells were seeded in $10 \mathrm{ml}$ complete media in a $10 \mathrm{~cm}$ dish. After 7 days, foci were stained with a $0.2 \%$ crystal violet solution ( $2 \%$ methanol). The total number of foci and individual foci size were quantified using ImageJ program. Data are expressed as means \pm SEM of three independent experiments performed in triplicate.

Anchorage-independent growth assay (soft agar assay): Assays were performed as previously described ${ }^{47-49}$. Briefly, cells were cultured in 12-well plates containing two layers of agar. Cells $\left(6 \times 10^{3}\right)$ were resuspended in $0.5 \%$ agar diluted in complete medium and poured onto a $1 \%$ layer of agar (diluted in medium). Fresh medium was added to the top layer every 3 days. After 2 weeks, colonies were stained with MTT, pictures were taken using a dissecting microscope, and colonies were counted using ImageJ software. Numbers are expressed as means \pm SEM of three independent experiments performed in triplicate.

Tumour sphere forming assay: Cells were cultured at a density of $2 \times 10^{4} /$ $35 \mathrm{~mm}$ dishes in a stem cell-permissive media. In particular, cells were cultured for one week in DMEM/F12 medium supplemented with $1 \% \mathrm{~N}-2$ Supplement, $2 \%$ B27, $50 \mathrm{mg} / \mathrm{ml}$ of Penicillin-Streptomycin, glutamine (Gibco), 0.01\% Bovine Serum Albumin (BSA), $5 \mathrm{mg} / \mathrm{ml}$ of insulin (Sigma) and growth factors including $10 \mathrm{ng} / \mathrm{ml}$ of basic fibroblast growth factor (bFGF), $20 \mathrm{ng} / \mathrm{ml}$ of epidermal growth factor (EGF) and $10 \mathrm{ng} / \mathrm{ml}$ of hepatocyte growth factor (HGF; Peprotech). After one week, pictures of the whole dish were taken using a dissecting microscope, and spheres were counted using ImageJ software. Numbers are expressed as means \pm SEM of three independent experiments performed in triplicate.

In vivo tumorigenesis assays (xenografts in nude mice): For in vivo demethylation studies, xenografts were performed using Alb-R26 ${ }^{\text {Met }}$ HCC cells either untreated or pre-treated for $48 \mathrm{~h}$ with Decitabine $(0.3 \mu \mathrm{M})$. Cells $\left(5 \times 10^{6}\right)$ were then resuspended in a 1:1 Matrigel:PBS solution (Corning BV) and inoculated subcutaneously into the flank-leg region of nude mice (S/SOPF SWISS NU/NU; Charles River). After 5 days of cell inoculation, mice were treated with intraperitoneal injections of vehicle or Decitabine $(2.5 \mathrm{mg} / \mathrm{kg})$ twice per week for 3 weeks. Mice were then sacrificed and tumour volume was measured as length $\times$ width $\times$ height. For assessment of in vivo tumorigenic capacity of candidate genes, xenografts were performed using Alb-R26 ${ }^{\text {Met }}$ HCC cells $\left(1 \times 10^{6}\right)$ either untransfected, transfected with shControl, or with a shRNA sequence targeting the candidate gene. Tumour volume was followed every week. After 6 weeks mice were sacrificed and tumour volume after dissection was determined as length $\times$ width $\times$ height.

Western blots: Protein extracts from HCC cells were prepared and western blot analysis was performed as previously described ${ }^{43,48,49}$. For SCN8A detection, protein lysates were run on a 5\% SDS gel and transferred overnight at $300 \mathrm{~mA}$ in the presence of $0.1 \%$ SDS. The acquisition of ECL signal was performed using the MyECL imager system (ThermoFisher Scientific)(Supplementary Fig. 15).

Antibodies: Antibodies used were: anti-SCN8A (Abcam, \#ab65166; 1:500), anti ACTN-1 (Cell Signalling, \#6487; 1:3000), anti-SRD5A2 (ThermoFisher Scientific, \#PA5-25465; 1:1000), anti-NFkB2 (Cell Signalling; \#4882; 1:1500), anti-NEURL1B (Abcam, \#ab156988; 1:3000), anti-ACTIN (Sigma, A3853; 1:5000), anti-rabbit IgGperoxidase or anti-mouse IgG-peroxidase (Jackson; 1:4000).

Computational analyses. Unsupervised hierarchical clustering analysis: Clustering statistics was determined by using the methylation values of all CGIs for each sample. We applied the Principal Component Analysis and the Agglomerative Distance Tree using the "linkage" function with unweighted average euclidean distance for calculating the similarity matrix of samples and the "dendrogram", as well as "phylotree" function to plot the hierarchical and distant trees (both are from Matlab Statistical Toolbox). For studies reported in Supplementary Fig. 7, clustering analysis of both methylome and expression data was performed using the function "hclust" on an Euclidean distance matrix of samples, which was computed with the function "dist". "hclust" then returned a tree-like structured object that could be plotted as dendrogram by "plot" (R, version 3.3.1).

Identification of human CGIs corresponding to the mouse CGIs of interest: To compare methylome outcomes identified in the Alb-R26 $6^{\text {Met }}$ genetic system with those available for human studies, genomic coordinates were converted from $\mathrm{mm} 9$ to GRCh37/hg19 by using the "Lift-Over" tool available from UCSC (https:// genome-euro.ucsc.edu/cgi-bin/hgLiftOver). This allowed us to successfully map 501 out of 513 CGIs from mouse to human regions (Supplementary Data 2). Among them, we only kept 501 unique human regions by discarding duplicate liftovers. We also discarded 14 human regions not overlapping with any human CGI We then check into TCGA patient datasets the presence of methylation data for those CGIs. We focused the analysis on the patient having both tumour and control samples (adjacent liver) methylation data, and we discarded the CGIs having no entry into any of the TCGA patient dataset. Finally, the total CGIs used for comparative analyses between mouse and human is 416 .

Analysis of public available DNA methylome data: The human methylome data is available through firebrowse (www.firebrowse.org) by the BROAD Institute and is based upon data generated by the TCGA Research Network: http:// cancergenome.nih.gov/. The publicly available methylome data (Level 3 data) of HCC patients from TCGA is generated with the platform Illumina Infinium Human DNA Methylation 450 and contains beta values for $485778 \mathrm{CpGs}$. Patients with both tumour and control samples were extracted and calculation of methylation difference per $\mathrm{CpG}$ was applied ( $\beta$-values of tumour- $\beta$-values of control). Student's $T$-test was used to compare between tumour and normal samples, and the $P$-values were corrected with Benjamini-Hochberg False Discovery Rate (FDR). As our methylome screen focused on CGIs, we revisited the human data (from TCGA and from GSE56588) to generate a list of all CpGs within CGIs with the corresponding methylation $\beta$-values. By applying the same methylation difference and FDR thresholds used for Alb-R26 $6^{\mathrm{Met}}$ methylome data, we extracted a list of differentially methylated CpGs from the human HCC dataset.

Methylome overlap between Alb-R26 $6^{\text {Met }}$ outcomes and human data: A methylation overlap between Alb-R26 $6^{\text {Met }}$ and human HCC was considered only when a given CGI was differentially methylated in both species. To define the methylation status of a given CGI, the CpG with an absolute maximum methylation difference among all patient samples was chosen as a representative probe (with $P$-value threshold and fold change cut-off defined above). This CpG was analysed in all HCC patients. An overlap score (in percentage) was determined by calculating the number of human CGIs differentially methylated versus the total number of lifted-over CGIs subset.

Analysis of public available RNA-seq data: The human RNA-seq data from TCGA was available through firebrowse. The data is generated with the platform Illumina HiSeq 2000 Sequencing System and uses MapSplice ${ }^{50}$ to do the alignment and RSEM ${ }^{51}$ to perform the quantitation. The scaled estimate from RSEM output was used as this value could be multiplied by $10^{6}$ to obtain a measure in terms of transcripts per million (TPM), which is preferred over RPKM ${ }^{52}$ or FPKM ${ }^{53}$ as it is independent of the mean transcript length and therefore more comparable across samples ${ }^{51}$. The TPM is calculated for each gene and the calculation of $\log _{2}$ Fold Change ( $\log _{2}$ (tumour sample) $-\log _{2}$ (control sample)) was applied to each patient with available data from both tumour and control samples.

Calculation of the relative position to the ATG: For calculating the position of CpGs, we used the longest transcript for each gene. The gene length was reported with values ranging from $-100 \%$ and $+100 \%$ (transcription end site: TES), with the ATG at position 0 . The relative position for each $\mathrm{CpG}$ was then reported 
relative to its distance to the ATG. A positive relative position corresponds to a genomic region located downstream the ATG, whereas a negative relative position stands for a genomic region located upstream the ATG.

Analysis of public available data from a mouse HCC model carrying the viral hepatitis $\mathrm{B}$ virus $\mathrm{X}$ expression: Using available methylome and expression data based on a HCC model induced by the viral hepatitis B virus $\mathrm{X}\left(H B x^{t g}\right.$; GSE48052 27 ), we performed the same analysis done for the Alb-R26 $6^{\text {Met }}$ model (Fig. 3b). For each $\mathrm{CpG}$, the methylation difference between $H B x^{t g}$ tumour and control sample was calculated as the difference of the RPKM. For those CpGs found differentially methylated, the expression of the corresponding gene was then calculated as the difference of the RPKM sum within the TSS and TSE.

Pathway enrichment analysis: For these analyses (shown in Supplementary Figs. 5, 9), identified genes were used as an input for KEGG pathway enrichment analysis with the REST API tool (http://rest.kegg.jp). Pathways were further ranked by $-\log _{10} P$-value after applying the hypergeometric probability density function (Matlab function "hygepdf" from Statistical Toolbox).

Statistical analysis: All data were analysed using GraphPad Prism software (version 7.01) and Matlab Statistical Toolbox (version R2015b). Results are expressed as the median (indicated by a line) or as the mean \pm standard error of the mean (SEM), according to sample distributions. Statistically significant differences were estimated by applying unpaired Student $t$-tests to data showing normal distributions, and Mann-Whitney tests in all other situations. Moreover, one-wayANOVA was used to determine differences between the means of independent groups (in vivo xenograft experiments in Figs. $2 \mathrm{~h}$ and $7 \mathrm{~d}$ ), and Fisher's exact test for categorical variables (risk factors in Supplementary Fig. 9). All statistical tests were two-sided. Statistical significance was defined as not significant (ns): $P>0.05$; ${ }^{*} P<0.05 ;{ }^{*} P<0.01 ;{ }^{* *} P<0.001$. Significance is indicated in figures

Data availability. Raw and processed data of bisulfite sequencing have been deposited to the Gene Expression Omnibus (GEO) [GEO: GSE90093]. The authors declare that all data supporting the findings of this study are available within the article and its Supplementary Information files, or from the authors upon reasonable request.

Received: 1 November 2017 Accepted: 10 July 2018

Published online: 08 August 2018

\section{References}

1. Esteller, M. Epigenetic changes in cancer. F1000 Biol. Rep. 3, 9 (2011).

2. Baylin, S. B. \& Jones, P. A. A decade of exploring the cancer epigenomebiological and translational implications. Nat. Rev. Cancer 11, 726-734 (2011).

3. Dawson, M. A. \& Kouzarides, T. Cancer epigenetics: from mechanism to therapy. Cell 150, 12-27 (2012).

4. Laird, P. W. Principles and challenges of genomewide DNA methylation analysis. Nat. Rev. Genet. 11, 191-203 (2010).

5. Ball, M. P. et al. Targeted and genome-scale strategies reveal gene-body methylation signatures in human cells. Nat. Biotechnol. 27, 361-368 (2009).

6. Woo, H. G. et al. Integrative analysis of genomic and epigenomic regulation of the transcriptome in liver cancer. Nat. Commun. 8, 839 (2017).

7. Feinberg, A. P. \& Tycko, B. The history of cancer epigenetics. Nat. Rev. Cancer 4, 143-153 (2004).

8. Jones, P. A. \& Baylin, S. B. The epigenomics of cancer. Cell 128, 683-692 (2007).

9. Jones, P. A. Functions of DNA methylation: islands, start sites, gene bodies and beyond. Nat. Rev. Genet. 13, 484-492 (2012).

10. Murtha, M. \& Esteller, M. Extraordinary cancer epigenomics: thinking outside the classical coding and promoter box. Trends Cancer 2, 572-584 (2016).

11. Heyn, H. et al. Epigenomic analysis detects aberrant super-enhancer DNA methylation in human cancer. Genome Biol. 17, 11 (2016).

12. Yang, X. et al. Gene body methylation can alter gene expression and is a therapeutic target in cancer. Cancer Cell 26, 577-590 (2014).

13. Jjingo, D., Conley, A. B., Yi, S. V., Lunyak, V. V. \& Jordan, I. K. On the presence and role of human gene-body DNA methylation. Oncotarget 3, 462-474 (2012).

14. Spencer, D. H. et al. CpG island hypermethylation mediated by DNMT3A is a consequence of AML progression. Cell 168, 801-816 e813 (2017).

15. Bender, S. et al. Reduced H3K27me3 and DNA hypomethylation are major drivers of gene expression in K27M mutant pediatric high-grade gliomas. Cancer Cell 24, 660-672 (2013).

16. Lu, C. et al. Histone H3K36 mutations promote sarcomagenesis through altered histone methylation landscape. Science 352, 844-849 (2016).

17. Lennartsson, A. External signals shape the epigenome. Genome Biol. 17, 18 (2016).

18. Liu, F., Wang, L., Perna, F. \& Nimer, S. D. Beyond transcription factors: how oncogenic signalling reshapes the epigenetic landscape. Nat. Rev. Cancer 16, 359-372 (2016).
19. Yao, X., Xing, M., Ooi, W. F., Tan, P. \& Teh, B. T. Epigenomic consequences of coding and noncoding driver mutations. Trends Cancer 2, 585-605 (2016).

20. Liu, F. et al. EGFR mutation promotes glioblastoma through epigenome and transcription factor network remodeling. Mol. Cell 60, 307-318 (2015).

21. Forloni, M. et al. Oncogenic EGFR represses the TET1 DNA demethylase to induce silencing of tumor suppressors in cancer cells. Cell Rep. 16, 457-471 (2016).

22. Nabet, B. et al. Deregulation of the Ras-Erk signaling axis modulates the enhancer landscape. Cell Rep. 12, 1300-1313 (2015).

23. Spangle, J. M. et al. PI3K/AKT signaling regulates $\mathrm{H} 3 \mathrm{~K} 4$ methylation in breast cancer. Cell Rep. 15, 2692-2704 (2016).

24. Fan, Y. et al. A Phosphokinome-based screen uncovers new drug synergies for cancer driven by liver-specific gain of non-oncogenic RTKs. Hepatology 66, 1644-1661 (2017).

25. Cancer Genome Atlas Research, N. et al. The cancer genome atlas Pan-cancer analysis project. Nat. Genet. 45, 1113-1120 (2013).

26. Villanueva, A. et al. DNA methylation-based prognosis and epidrivers in hepatocellular carcinoma. Hepatology 61, 1945-1956 (2015).

27. Lee, S. M. et al. HBx induces hypomethylation of distal intragenic CpG islands required for active expression of developmental regulators. Proc. Natl Acad. Sci. USA 111, 9555-9560 (2014).

28. Zucman-Rossi, J., Villanueva, A., Nault, J. C. \& Llovet, J. M. Genetic landscape and biomarkers of hepatocellular carcinoma. Gastroenterology 149, 1226-1239 e1224 (2015).

29. Azad, N., Zahnow, C. A., Rudin, C. M. \& Baylin, S. B. The future of epigenetic therapy in solid tumours--lessons from the past. Nat. Rev. Clin. Oncol. 10, 256-266 (2013).

30. Brien, G. L., Valerio, D. G. \& Armstrong, S. A. Exploiting the epigenome to control cancer-promoting gene-expression programs. Cancer Cell 29, 464-476 (2016).

31. Bhasin, J. M. et al. Methylome-wide sequencing detects DNA hypermethylation distinguishing indolent from aggressive prostate cancer. Cell Rep. 13, 2135-2146 (2015).

32. Kulis, M. et al. Epigenomic analysis detects widespread gene-body DNA hypomethylation in chronic lymphocytic leukemia. Nat. Genet. 44, 1236-1242 (2012).

33. Lister, R. et al. Human DNA methylomes at base resolution show widespread epigenomic differences. Nature 462, 315-322 (2009).

34. Varley, K. E. et al. Dynamic DNA methylation across diverse human cell lines and tissues. Genome Res. 23, 555-567 (2013).

35. Maunakea, A. K. et al. Conserved role of intragenic DNA methylation in regulating alternative promoters. Nature 466, 253-257 (2010).

36. Hahn, M. A., Wu, X., Li, A. X., Hahn, T. \& Pfeifer, G. P. Relationship between gene body DNA methylation and intragenic $\mathrm{H} 3 \mathrm{~K} 9 \mathrm{me} 3$ and $\mathrm{H} 3 \mathrm{~K} 36 \mathrm{me} 3$ chromatin marks. PLoS ONE 6, el8844 (2011).

37. Karch, F. In vivo studies of the Drosophila insulator factor CTCF reach a Catch 22. BMC Biol. 13, 71 (2015).

38. Shukla, S. et al. CTCF-promoted RNA polymerase II pausing links DNA methylation to splicing. Nature 479, 74-79 (2011).

39. Hon, G. C. et al. Global DNA hypomethylation coupled to repressive chromatin domain formation and gene silencing in breast cancer. Genome Res. 22, 246-258 (2012).

40. Maina, F. Strategies to overcome drug resistance of receptor tyrosine kinaseaddicted cancer cells Curr. Med. Chem. 21, 1607-1617 (2014).

41. Scott, M. T. et al. Epigenetic reprogramming sensitizes CML stem cells to combined EZH2 and tyrosine kinase inhibition. Cancer Discov. 6, 1248-1257 (2016).

42. Genestine, M. et al. Enhanced neuronal Met signalling levels in ALS mice delay disease onset. Cell Death Dis. 2, e130 (2011).

43. Fan, Y. et al. Tissue-specific gain of RTK signalling uncovers selective cell vulnerability during embryogenesis. PLoS Genet. 11, el005533 (2015).

44. Krueger, F. \& Andrews, S. R. Bismark: a flexible aligner and methylation caller for Bisulfite-Seq applications. Bioinformatics 27, 1571-1572 (2011).

45. Dobin, A. et al. STAR: ultrafast universal RNA-seq aligner. Bioinformatics 29, 15-21 (2013).

46. Trapnell, C. et al. Differential gene and transcript expression analysis of RNAseq experiments with TopHat and Cufflinks. Nat. Protoc. 7, 562-578 (2012).

47. Furlan, A. et al. Met acts through Abl to regulate p53 transcriptional outcomes and cell survival in the developing liver. J. Hepatol. 57, 1292-1298 (2012).

48. Furlan, A. et al. Combined drug action of 2-phenylimidazo[2,1-b] benzothiazole derivatives on cancer cells according to their oncogenic molecular signatures. PLoS ONE 7, e46738 (2012).

49. Furlan, A. et al. Abl interconnects oncogenic Met and p53 core pathways in cancer cells. Cell Death Differ. 18, 1608-1616 (2011).

50. Wang, K. et al. MapSplice: accurate mapping of RNA-seq reads for splice junction discovery. Nucleic Acids Res. 38, el78 (2010).

51. Li, B., Ruotti, V., Stewart, R. M., Thomson, J. A. \& Dewey, C. N. RNA-Seq gene expression estimation with read mapping uncertainty. Bioinformatics $\mathbf{2 6}$, 493-500 (2010). 
52. Mortazavi, A., Williams, B. A., McCue, K., Schaeffer, L. \& Wold, B. Mapping and quantifying mammalian transcriptomes by RNA-Seq. Nat. Methods $\mathbf{5}$, 621-628 (2008).

53. Trapnell, C. et al. Transcript assembly and quantification by RNA-Seq reveals unannotated transcripts and isoform switching during cell differentiation. Nat. Biotechnol. 28, 511-515 (2010).

\section{Acknowledgements}

These results are in part based upon public data generated by TCGA Research Network: http://cancergenome.nih.gov/. We are particularly grateful to F. Helmbacher for extremely valuable feedback on the study. We thank: all members of our labs for helpful discussions and comments; F. Castets for advises on biochemical analysis; J. ZucmanRossi and $\mathrm{E}$. Letouzé for valuable discussion and feedback on the bioinformatics; $\mathrm{V}$. Girod-David and L. Jullien for excellent help with mouse husbandry; C. Giaccherini, A. Dobric and E. Chrabaszcz for their contributions to studies on molecular and functional characterisations of shRNA-targeted HCC cells. This work was funded by INCa (Institut National du Cancer), FdF (Fondation de France) and GEFLUC-Les Entreprises contre le Cancer to F.M. M.A. was supported by a FdF fellowship. A.Y. was supported by DFG grant HA 6905/2-1. S.K.B. was supported by the Higher Education Commission (HEC) of Pakistan-France Campus. The contribution of the Region Provence Alpes Côtes d'Azur and of the Aix-Marseille Université to the IBDM animal facility is also acknowledged. The funders had no role in study design, data collection and analysis, decision to publish or preparation of the manuscript.

\section{Author contributions}

M.A.: performed the majority of the experiments, data analysis, interpretation, contributed to computational work and writing the paper. F.D.: performed the majority of the computational work, data analysis and interpretation. A.Y.: performed computational work using the human HCC cohort from TCGA database, data analysis and interpretation. S.K.B.: contributed to molecular and functional studies with HCC cells. S.R.: prepared tumour samples and genomic DNA for methylome; contributed to xenograft studies. R.D.: contributed to establishing the mouse model, to interpreting data and provided input on writing the paper. A.J.S.: provided support for computational work, contributed to interpreting data and provided input on writing the paper. B.H.H.: analysed and interpreted transcriptome data from mouse model, supervised studies on human HCC data and provided input on writing the paper. F.M.: designed the study, performed experiments, analysed and interpreted data, ensured financial support and wrote the paper.

\section{Additional information}

Supplementary Information accompanies this paper at https://doi.org/10.1038/s41467 018-05550-5.

Competing interests: The authors declare no competing interests.

Reprints and permission information is available online at http://npg.nature.com/ reprintsandpermissions/

Publisher's note: Springer Nature remains neutral with regard to jurisdictional claims in published maps and institutional affiliations.

(c) (i) Open Access This article is licensed under a Creative Commons Attribution 4.0 International License, which permits use, sharing, adaptation, distribution and reproduction in any medium or format, as long as you give appropriate credit to the original author(s) and the source, provide a link to the Creative Commons license, and indicate if changes were made. The images or other third party material in this article are included in the article's Creative Commons license, unless indicated otherwise in a credit line to the material. If material is not included in the article's Creative Commons license and your intended use is not permitted by statutory regulation or exceeds the permitted use, you will need to obtain permission directly from the copyright holder. To view a copy of this license, visit http://creativecommons.org/ licenses/by/4.0/.

(C) The Author(s) 2018 